\title{
Simulations of direct and reflected wave trajectories for ground-based GNSS-R experiments
}

\author{
N. Roussel ${ }^{1,2}$, F. Frappart ${ }^{1,2}$, G. Ramillien ${ }^{1,2}$, J. Darrozes ${ }^{1,2}$, C. Desjardins ${ }^{1,2,3,4}$, P. Gegout ${ }^{1,2}$, F. Pérosanz ${ }^{1,2,4}$, and \\ R. Biancale B $^{1,2,4}$ \\ ${ }^{1}$ Université de Toulouse, CNRS, IRD, GET-OMP, Toulouse, France \\ ${ }^{2}$ Groupe de Recherche en Géodésie Spatiale, Toulouse, France \\ ${ }^{3}$ Collecte Localisation Satellites, Ramonville Saint Agne, France \\ ${ }^{4}$ Centre National d'Etudes Spatiales, Toulouse, France \\ Correspondence to: N. Roussel (nicolas.roussel@get.obs-mip.fr)
}

Received: 30 November 2013 - Published in Geosci. Model Dev. Discuss.: 24 January 2014

Revised: 12 August 2014 - Accepted: 31 August 2014 - Published: 2 October 2014

\begin{abstract}
The detection of Global Navigation Satellite System (GNSS) signals that are reflected off the surface, along with the reception of direct GNSS signals, offers a unique opportunity to monitor water level variations over land and ocean. The time delay between the reception of the direct and reflected signals gives access to the altitude of the receiver over the reflecting surface. The field of view of the receiver is highly dependent on both the orbits of the GNSS satellites and the configuration of the study site geometries. A simulator has been developed to determine the location of the reflection points on the surface accurately by modeling the trajectories of GNSS electromagnetic waves that are reflected by the surface of the Earth. Only the geometric problem was considered using a specular reflection assumption. The orbit of the GNSS constellation satellites (mainly GPS, GLONASS and Galileo), and the position of a fixed receiver, are used as inputs. Four different simulation modes are proposed, depending on the choice of the Earth surface model (local plane, osculating sphere or ellipsoid) and the consideration of topography likely to cause masking effects. Angular refraction effects derived from adaptive mapping functions are also taken into account. This simulator was developed to determine where the GNSS-R receivers should be located to monitor a given study area efficiently. In this study, two test sites were considered: the first one at the top of the $65 \mathrm{~m}$ Cordouan lighthouse in the Gironde estuary, France, and the second one on the shore of Lake Geneva ( $50 \mathrm{~m}$ above the reflecting surface), at the border between France and Switzerland. This site is hidden by mountains in the south (orthometric
\end{abstract}

altitude up to $2000 \mathrm{~m}$ ), and overlooking the lake in the north (orthometric altitude of $370 \mathrm{~m}$ ). For this second test site configuration, reflections occur until $560 \mathrm{~m}$ from the receiver. The planimetric (arc length) differences (or altimetric difference as WGS84 ellipsoid height) between the positions of the specular reflection points obtained considering the Earth's surface as an osculating sphere or as an ellipsoid were found to be on average $9 \mathrm{~cm}$ (or less than $1 \mathrm{~mm}$ ) for satellite elevation angles greater than $10^{\circ}$, and $13.9 \mathrm{~cm}$ (or less than $1 \mathrm{~mm}$ ) for satellite elevation angles between 5 and $10^{\circ}$. The altimetric and planimetric differences between the plane and sphere approximations are on average below $1.4 \mathrm{~cm}$ (or less than $1 \mathrm{~mm}$ ) for satellite elevation angles greater than $10^{\circ}$ and below $6.2 \mathrm{~cm}$ (or $2.4 \mathrm{~mm}$ ) for satellite elevation angles between 5 and $10^{\circ}$. These results are the means of the differences obtained during a $24 \mathrm{~h}$ simulation with a complete GPS and GLONASS constellation, and thus depend on how the satellite elevation angle is sampled over the day of simulation. The simulations highlight the importance of the digital elevation model (DEM) integration: average planimetric differences (or altimetric) with and without integrating the DEM (with respect to the ellipsoid approximation) were found to be about $6.3 \mathrm{~m}$ (or $1.74 \mathrm{~m}$ ), with the minimum elevation angle equal to $5^{\circ}$. The correction of the angular refraction due to troposphere on the signal leads to planimetric (or altimetric) differences of an approximately $18 \mathrm{~m}$ (or $6 \mathrm{~cm}$ ) maximum for a $50 \mathrm{~m}$ receiver height above the reflecting surface, whereas the maximum is $2.9 \mathrm{~m}$ (or $7 \mathrm{~mm}$ ) for a $5 \mathrm{~m}$ receiver height above the reflecting surface. These errors 
increase deeply with the receiver height above the reflecting surface. By setting it to $300 \mathrm{~m}$, the planimetric errors reach $116 \mathrm{~m}$, and the altimetric errors reach $32 \mathrm{~cm}$ for satellite elevation angles lower than $10^{\circ}$. The tests performed with the simulator presented in this paper highlight the importance of the choice of the Earth's representation and also the nonnegligible effect of angular refraction due to the troposphere on the specular reflection point positions. Various outputs (time-varying reflection point coordinates, satellite positions and ground paths, wave trajectories, first Fresnel zones, etc.) are provided either as text or KML files for visualization with Google Earth.

\section{Introduction}

The Global Navigation Satellite System (GNSS), which includes the American GPS, the Russian GLONASS, and the European Galileo (which is getting denser), uses L-band microwave signals to provide accurate 3-D positioning on any point of the Earth's surface or close vicinity. Along with the space segment development, the processing techniques have also improved considerably, with a better consideration of the various sources of error in the processing. Among them, multipaths still remain a major problem, and the mitigation of their influence has been widely investigated (Bilich, 2004). The ESA (European Space Agency) first proposed the idea of taking advantage of the multipath phenomenon in order to assess different parameters of the reflecting surface (MartinNeira, 1993). This opportunistic remote sensing technique, known as GNSS reflectometry (GNSS-R), is based on the analysis of the electromagnetic signals emitted continuously by the GNSS satellites and detected by a receiver after reflection on the Earth's surface. Several parameters of the Earth's surface can be retrieved either by using the time delay between the signals received by the upper (direct signal) and lower (reflected signal) antennas, or by analyzing the waveforms (temporal evolution of the signal power) corresponding to the reflected signal. This technique offers a wide range of applications in Earth sciences. The time delay can be interpreted in terms of altimetry as the difference in height between the receiver and the surface. Temporal variations of sea (Lowe et al., 2002; Ruffini et al., 2004; Löfgren et al., 2011; Semmling et al., 2011; Rius et al., 2012) and lake levels (Treuhaft et al., 2004; Helm, 2008) were recorded with an accuracy of a few $\mathrm{cm}$ using in situ and airborne antennas. Surface roughness can be estimated from the analysis of the delay Doppler maps (DDM) derived from the waveforms of the reflected signals. They can be related to parameters such as soil moisture (Katzberg et al., 2006; RodriguezAlvarez et al., 2009, 2011) over land, wave heights and wind speed (Komjathy et al., 2000; Zavorotny and Voronovich, 2000; Rius et al., 2002; Soulat et al., 2004) over the ocean, or ice properties (Gleason, 2006; Cardellach et al., 2012). The GNSS-R technique presents two main advantages: (1) a dense spatial and temporal coverage, not only limited to a single measurement point or a non-repetitive transect as with using classical GNSS buoys, and (2) a guarantee of service for the next decades (because of the strategic role played by these systems). GNSS-R altimetric accuracy is today at the level of a few $\mathrm{cm}$, but this technique will benefit, in the future, from improved processing techniques and from the densification of the GNSS constellation. The commonly used GNSS-R system consists of two antennas (Fig. 1): the first one is right-hand circular polarized (RHCP) and zenith facing to receive the direct waves. The second one is left-hand circular polarized (LHCP) and nadir facing to receive the reflected waves. These reflected waves will predominantly change their polarization from RCHP to LHCP by reflecting at near-normal incidence. The reflected signals have an additional path delay with respect to the direct ones. The analysis of the path difference between these direct and reflected signals is used to estimate the relative height difference between the two antennas. In order to anticipate the impact of the geometric configuration of the experiment, a simulator has been developed to estimate the positions of reflection points using a specular reflection point assumption. Four different methods were implemented: approximating the Earth's surface as a local plane, as an osculating sphere, as an ellipsoid, or integrating a digital elevation model (DEM). In addition, the signal bending due to the neutral part of the atmosphere is taken into account using the adaptive mapping functions (AMF) from Gégout et al. (2011), and made available by GRGS (Groupe de Recherche en Géodésie Spatiale). Simulations were performed for different configurations: variations in the reflectometer height, mask effects due to terrain, and satellite network geometry.

This article is composed of three main parts following the logical structure of Fig. 2. The first part presents the data sets used for initiating simulations, the second one concerns the methodologies for the determination of the reflection points, while the last one deals with the simulator performances and simulation results.

\section{Design of the simulator}

The simulator has been developed in the GNU R language, generally used for data processing and statistical analysis. A user manual and a description of the $\mathrm{R}$ language can be found on the website http://www.r-project.org/. The main interest of such a language remains in that it is distributed under a GNU GPL license that does $\mathrm{R}$ routines in an open source program, available on various platforms (i.e., GNU/Linux, FreeBSD, NetBSD, OpenBSD, Mac OS and Windows).

The simulator is composed of three main blocks (Fig. 2): an input block that contains the different elements mandatory for the processing, a processing block where the user can choose which algorithm to use, and an output block containing the different results of the simulation. 


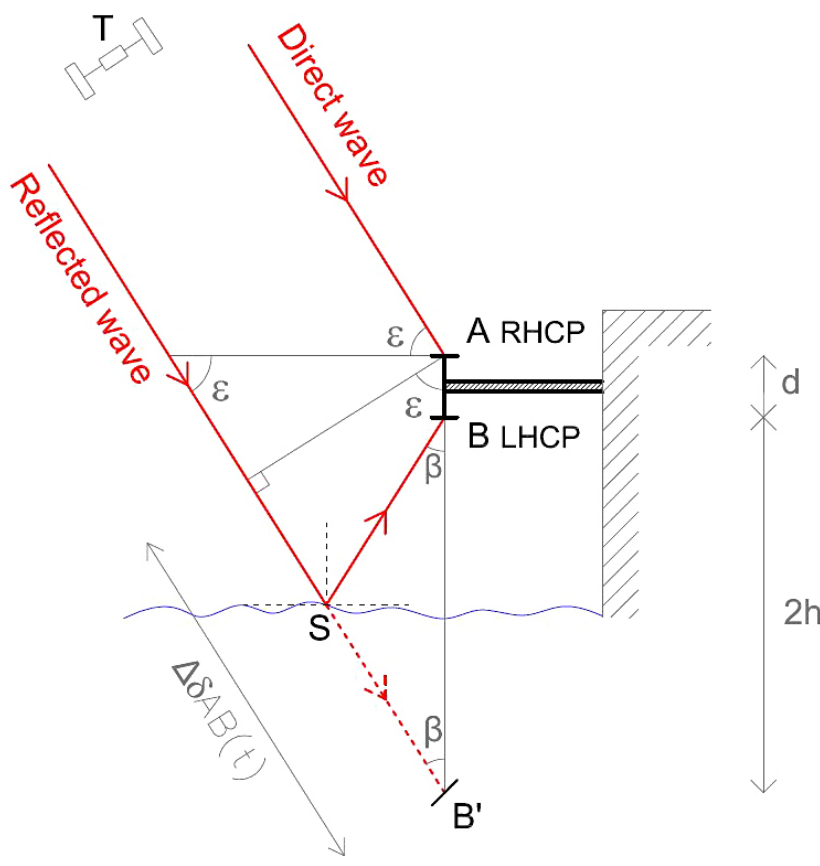

Figure 1. Principle of GNSS reflectometry. $T$ : satellite/transmitter; $S$ : specular reflection point; $\epsilon$ : satellite elevation; $\triangle \delta \mathrm{AB}(t)$ : additional path covered by the reflected wave; $d$ : interdistance between the LHCP and RHCP antennas; and $h$ : height of the receiver above the reflecting surface.

As inputs, this simulator requires the receiver coordinates, the satellite ephemeris and a set of optional environmental parameters such as a DEM in order to take the possible masking of the terrestrial topography into account, as well as adaptive mapping functions to integrate atmospheric delays and bending effects.

As outputs, the simulator provides the time-varying reflection point coordinates, but also various KML (Keyhole Markup Language - the standard format used by Google Earth) files such as satellite positions and ground paths, wave trajectories and Fresnel first surfaces that can be opened using the Google Earth visualization tool.

\section{Data sets}

\subsection{GNSS orbit parameters}

The simulations are based on the determination of the positions of the specular reflection points, once the receiver and the satellite positions are known. Satellite coordinates can be obtained from the International GNSS Service (IGS) ephemeris final products, which provide GNSS orbit and clock offset data with a temporal resolution of $15 \mathrm{~min}$ in the SP3 format for the past epochs, or are derived from the Keplerian parameters (semi-major axis, inclination, and argument of perigee) to predict GNSS satellite

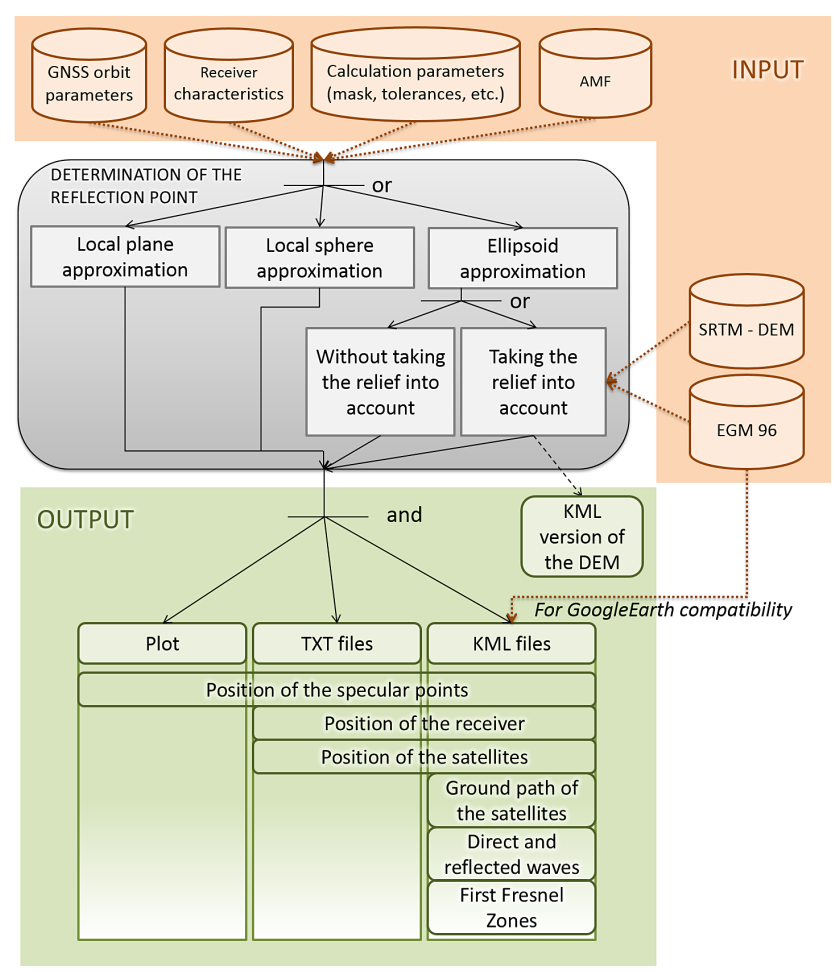

Figure 2. Data flowchart of the simulator. Three main blocks: an input block that contains the different elements mandatory for the processing, a processing block where the user can choose which algorithm is to be used, and an output block containing the different results of the simulation, namely KML files to be opened with Google Earth.

positions. Ephemeris products are available on the IGS website (http://igs.org/), and Keplerian parameters, e.g., at http: //www.navcen.uscg.gov.

\subsection{Radio-electric mask}

Simulations are performed for a given receiver position in the WGS84 coordinate system and height above the ground. It is possible to apply an elevation or azimuthal angle mask to the simulations to avoid satellites with low elevation angles, for instance. The elevation angle mask commonly used is set to a $10^{\circ}$ minimum and a $90^{\circ}$ maximum, and no mask is set in the azimuth.

\subsection{SRTM digital elevation model}

The most realistic simulation needs the integration of a digital elevation model (DEM) in order not only to take the possible masking of satellites into account, but to get more accurate and exact positions of the specular reflection points as well. The hole-filled version 4 of the Shuttle Radar Topography Mission (SRTM) DEM, with a spatial resolution of $90 \mathrm{~m}$ at the Equator, is used (Jarvis et al., 2008). The altitudes are given with reference to the EGM96 geoid model. Uncertainty 
in altitude is around $16 \mathrm{~m}$ over mountainous areas (Rodriguez et al., 2005). It is made available by files of $5^{\circ} \times 5^{\circ}$ for land areas between $60^{\circ} \mathrm{N}$ and $60^{\circ} \mathrm{S}$ by the Consortium for Spatial Information (CGIAR-CSI; http://srtm.csi.cgiar.org/).

\subsection{EGM96 Earth gravitational model}

In order to be able to convert between ellipsoidal heights (with respect to the WGS84 ellipsoid) and altitudes (with respect to the EGM96 geoid model) when producing KML files or when integrating a DEM, knowledge of the geoid undulation is mandatory. In this study, we interpolate a $15 \times 15 \mathrm{~min}$ geoid undulation grid file derived from the EGM96 model in a tide-free system released by the US National GeospatialIntelligence Agency (NGA) EGM development team (http:// earth-info.nga.mil/GandG/wgs84/gravitymod/). The error in the interpolation is lower than $2 \mathrm{~cm}$ (NASA and NIMA, 1998).

\subsection{Adaptive mapping functions}

The neutral atmosphere bends the propagation path of the GNSS signal and retards the speed of propagation. The range between the satellite and the tracking site is neither the geometric distance nor the length of the propagation path, but the radio range of the propagation path (Marini, 1972).

For GNSS-R measurements, the tropospheric effects induced by the neutral part of the atmosphere are an important source of error. Indeed, GNSS-R measurements are often made at low elevation angles, where the bending effects are maximal. Accurate models have to be used to mitigate signal speed decrease and path bending. Modeling tropospheric delays by calculating the zenith tropospheric delay and obtaining the slant tropospheric delays with a mapping function is commonly accepted. New mapping functions were developed in the 2000s (Boehm et al., 2006a; Niell, 2001), and significantly improve the geodetic positioning. Although modern mapping functions like VMF1 (Boehm et al., 2006b) and GPT2/VMF1 (Lagler et al., 2013) are derived from numerical weather models (NWM), most of these mapping functions ignore the azimuth dependency, which is usually introduced by two horizontal gradient parameters - in the northsouth and east-west directions - estimated directly from observations (Chen et al., 1997). More recently, the use of raytraced delays through NWM directly at observation level has shown an improvement in geodetic results (Hobiger et al., 2008; Nafisi et al., 2012; Zus et al., 2012). The adaptive mapping functions (AMF) are designed to fit most of the information available in NWM - especially the azimuth dependency - preserving the classical mapping function strategy. AMF are thus used to approximate thousands of atmospheric raytraced delays using a few tens of coefficients with millimeter accuracy at low elevations (Gégout et al., 2011). AMF have a classical form, with terms that are functions of the elevation, but they also include coefficients that depend on the

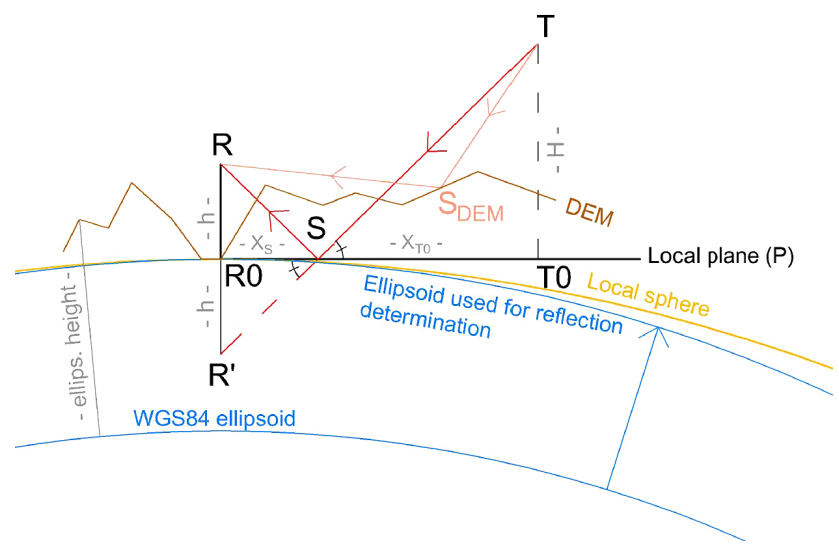

Figure 3. Determination of the specular reflection point in a local plane approximation and local difference with the sphere and ellipsoid approximations and DEM integration. $S$ : specular reflection point position. $R$ : receiver position. $T$ : transmitter/satellite position. $h$ : height of the receiver above the ground surface.

azimuth to represent the azimuthal dependency of ray-traced delays. In addition, AMF are suitable for adapting to complex weather by changing the truncation of the successive fractions. Therefore, the AMF are especially suited to correcting the propagation of low-elevation GNSS-R signals. In our study, we use AMF directly provided by GRGS (Groupe de Recherche en Géodésie Spatiale) and computed following Gégout et al. (2011).

\subsection{Data used for a simulator usage illustration}

In order to assess the ocean tide influence on the positions of the reflection points estimated at an offshore experimental site located at the top of the Cordouan lighthouse $\left(45^{\circ} 35^{\prime} 11^{\prime \prime} \mathrm{N} ; 1^{\circ} 10^{\prime} 24^{\prime \prime} \mathrm{W}\right)$, we use $24 \mathrm{~h}$ of REFMAR (Réseau de Référence des Observations Marégraphiques) tide gauge observations, with a sampling frequency of 5 minutes. The tide gauge records of the station of Royan $\left(45^{\circ} 37^{\prime} 14.07^{\prime \prime} \mathrm{N} ; 1^{\circ} 01^{\prime} 40.12^{\prime \prime}\right.$, located $12 \mathrm{~km}$ from the lighthouse) are the property of MEDDE (Ministère de l'Ecologie, du Développement Durable et de l'Energie), and they are available on the REFMAR website (http://refmar.shom.fr).

\section{Methodology: determination of the positions of reflection points}

The difference in phase between the two antennas (A-RHCP and B-LHCP in Fig. 1) at an epoch $t$ for the $i$ th GNSS satellite can be seen as a classical single difference between two receivers used for relative positioning as follows:

$\lambda \Delta \phi_{\mathrm{AB}}^{i}(t)=\Delta \delta_{\mathrm{AB}}^{i}(t)-\lambda \Delta N_{\mathrm{AB}}^{i}-c \Delta t_{\mathrm{AB}}$,

where $\lambda$ is the wavelength of the GNSS signal, $\Delta \phi_{\mathrm{AB}}^{i}$ the measured carrier phase difference between the direct and 
received signals expressed in cycles, $\Delta \delta_{\mathrm{AB}}^{i}$ the difference in distance between the direct and received signals, $\Delta N_{\mathrm{AB}}^{i}$ is the difference of phase ambiguity between the direct and received signals, $c$ the speed of light in a vacuum, and $\Delta t_{\mathrm{AB}}$ the receiver clock bias difference. As the baseline between the two receivers is short (a few $\mathrm{cm}$ to a few tenths of $\mathrm{cm}$ ), and in the case of low altitude of the receivers, both tropospheric and ionospheric effects are neglected due to the spatial resolution of the current atmospheric and ionospheric models. Besides, when both antennas are connected to the same receiver, the receiver clock bias difference is also cancelled out. In this study, we only consider the difference in distance between direct and reflected signals, as illustrated in Fig. 1.

The processing block contains four algorithms for determining the positions of the specular reflection points: the first considering the Earth as a local plane in the vicinity of the reflection point, the second as an osculating sphere, the third as an ellipsoid that corresponds to the WGS84 ellipsoid, which has been expanded until the ellipsoid height of the receiver equals the height of the receiver above the reflecting surface (see Sect. 3.3), and the last one uses the ellipsoid approximation, but takes the Earth's topography into account: see Fig. 3. Comparisons between the different approximations of the Earth's shape will be performed in Sect. 4.1.

All of them are based on iterative approaches to solving the Snell-Descartes law for reflection: the unique assumption is that the angle of incidence is equal to the angle of reflection on a plane interface separating two half-space media (a locally planar approximation is adopted when the surface is not planar everywhere). In the plane, sphere and ellipsoid approximations, the specular reflection point of a given satellite is contained within the plane defined by the satellite, the receiver and the center of the Earth. With regards to the DEM integration, reflection can occur everywhere. In order to be able to compare the specular reflection point positions obtained by integrating a DEM, and to simplify the problem, we will only consider the reflections occurring within the plane, even while integrating a DEM.

\subsection{Local plane reflection approximation}

Refering to Fig. 3, let us consider the projection of the receiver $R 0$ on the osculating sphere approximation (see Sect. 3.2). We define the local plane $P$ as the plane tangent to the sphere at $R 0$. Let $T 0$ be the projection of the satellite on $P$ and $R^{\prime}$ the symmetry of $R 0$ relative to $P$. We look for the positions of the specular reflection points on $P$. Considering the Thales theorem in triangles $R^{\prime} S R 0$ and $S T T 0$, we have (see Fig. 3)

$\frac{X_{S}}{\left(X_{T 0}-X_{S}\right)}=\frac{h}{H}$.
Thus,

$X_{S}=\frac{h X_{T 0}}{H+h}$.

\subsection{Local sphere reflection approximation}

The model we consider is an osculating sphere. Its radial direction coincides with the ellipsoidal normal, and its center is set at an ellipsoidal height equal to the negative value of the Gaussian radius of curvature defined as

$r_{E}=\frac{a^{\prime 2} b^{\prime}}{a^{\prime 2} \cos ^{2}(\varphi)+b^{\prime 2} \sin ^{2}(\varphi)}$,

with $\varphi$ the latitude of the receiver, and $a^{\prime}$ and $b^{\prime}$ the semimajor and semi-minor axes of the modified ellipsoid (see Sect. 3.3). Please refer to Nievinski and Santos (2010) for further information on the different approximations of the Earth, particularly on the osculating sphere.

J. Kostelecky and C. Wagner already suggested an algorithm to retrieve the specular reflection point positions by approximating the Earth as a sphere (Kostelecky et al., 2005; Wagner and Klokocnik, 2003). Their algorithm is based on an optimized iterative scheme that is equivalent to making the position of a fictive specular point vary until verifying the first law of Snell and Descartes. A similar approach will be used in this paper in Sect. 3.3 with the ellipsoid approximation. Here, we chose to adopt a more analytical algorithm, first proposed by Helm (2008). In order to validate this algorithm, comparisons between it and the iterative one developed for the ellipsoid approach will be performed, by setting the minor and major axes of the ellipsoid equal to the sphere radius (see Sect. 4.2.1).

Let us consider the vertical plane formed by the transmitter (GNSS) satellite $(T)$, the receiver $(R)$ and $O^{\prime}$, the center of the Earth (Fig. 4). We assume that the specular reflection point $(S)$ will be included in that plane. Let us consider the following orthonormal reference systems of coordinates:

- $(O, X, Y, Z)_{R 1}:$ WGS84 Cartesian system (NIMA, 1997), with $O$ the center of the Earth. WGS84 has $Z$ polar and $X, Y$ equatorial. The receiver and transmitter coordinates are known in this system.

- $\left(O^{\prime}, x, y\right)_{R 2}$ : a local 2-D system, obtained by the rotation of the $(O, X, Y, Z)$ system around the $Z$ axis, in such a way that $x_{\mathrm{r}}$ equals 0 , and a translation $\mathbf{0 0}^{\prime}$ with $0^{\prime}$ the center of the osculating sphere.

- $\left(S, x^{\prime}, y^{\prime}\right)_{R 3}$ : a local 2-D system, obtained by a rotation around the $z$ axis and a $r_{E}$ translation of the $\left(O^{\prime}, x, y\right)$ system in such a way that $x^{\prime}$ and the local vertical are colinear, and the system origin coincides with the specular reflection point $S$. 


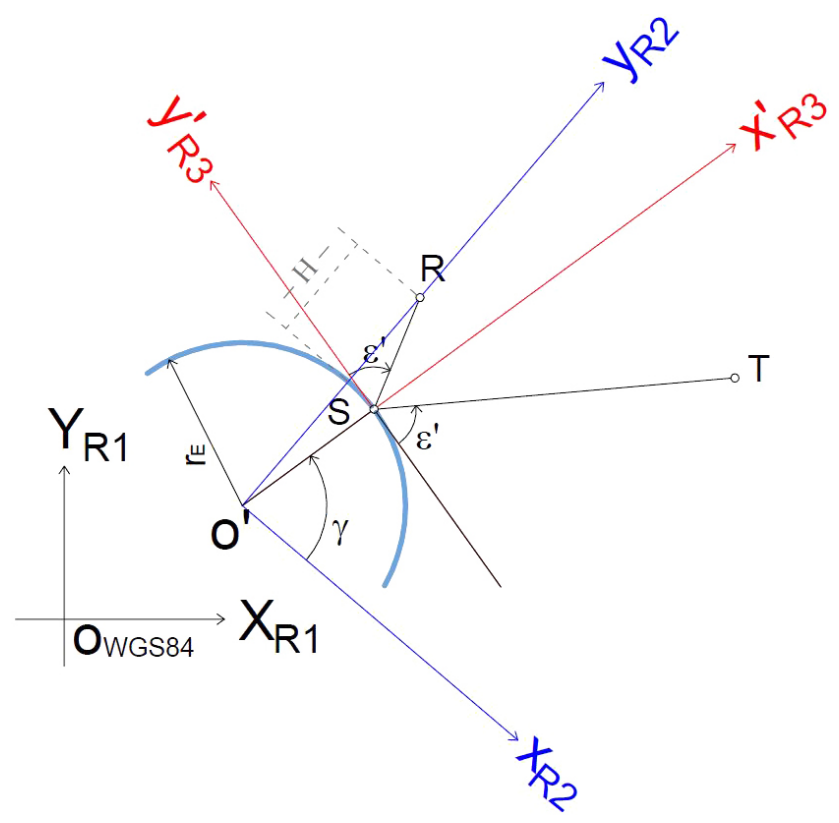

Figure 4. Local osculating sphere approximation: the three different reference systems of coordinates. $S$ : specular reflection point position. $R$ : receiver position. $T$ : transmitter/satellite position. $(0, X, Y, Z)_{R 1}$ : WGS84 Cartesian system. $\left(0^{\prime}, x, y\right)_{R 2}$ : local 2-D system, centred on the center of the osculating sphere, obtained by the rotation of the $R 1$ system around the $Z$ axis, in such a way that $x_{\mathrm{r}}$ equals $0 .\left(S, x^{\prime}, y^{\prime}\right)_{R 3}$ : a local 2-D system, obtained by a rotation around the $z$ axis and a $r_{R}$ translation of the R2 system in such a way that $x^{\prime}$ and the local vertical are colinear and the system origin coincides with the specular reflection point $S$.

If $H$ is the height of the receiver above the ground, the position of the receiver is

$\boldsymbol{r}_{\mathrm{r}}=\left(\begin{array}{l}x_{\mathrm{r}} \\ y_{\mathrm{r}}\end{array}\right)_{R 2}=\left(\begin{array}{c}0 \\ r_{E}+H\end{array}\right)_{R 2}$,

with $r_{E}$ the Gaussian radius of curvature at the latitude of the receiver $\varphi_{\mathrm{r}}$.

The position of the GNSS satellite transmitter considering $\varepsilon$ the elevation angle of the satellite (considering the zenith angle reckoned from the ellipsoidal normal direction) and $\tau$ the angle $\widehat{R T O^{\prime}}$ is given by

$\boldsymbol{r}_{t}=\left(\begin{array}{l}x_{t} \\ y_{t}\end{array}\right)_{R 2}=\left(\begin{array}{l}r_{t} \cos (\varepsilon+\tau) \\ r_{t} \sin (\varepsilon+\tau)\end{array}\right)_{R 2}$

Using the trigonometric sine formula in the $R-T-0^{\prime}$ triangle,

$\frac{\sin \left(\frac{\pi}{2}+\varepsilon\right)}{r_{t}}=\frac{\sin (\tau)}{r_{E}+H}$ we finally obtain

$$
\left(\begin{array}{c}
x_{t} \\
y_{t}
\end{array}\right)_{R 2}=\left(\begin{array}{c}
r_{t} \cos (\varepsilon) \sqrt{1-\frac{\left(r_{E}+H\right)^{2}}{r_{t}^{2}} \cos ^{2}(\varepsilon)} \\
-\left(r_{E}+H\right) \sin (\varepsilon) \cos (\varepsilon) \\
r_{t} \sin (\varepsilon) \sqrt{1-\frac{\left(r_{E}+H\right)^{2}}{r_{t}^{2}} \cos ^{2}(\varepsilon)} \\
-\left(r_{E}+H\right) \cos ^{2}(\varepsilon)
\end{array}\right)_{R 2} .
$$

The Snell-Descartes law for reflection can be expressed as the ratios of the coordinates of the receiver and the transmitter in $\left(S, x^{\prime}, y^{\prime}\right)$ :

$\frac{x_{t}^{\prime}}{y_{t}^{\prime}}=\frac{x_{\mathrm{r}}^{\prime}}{y_{\mathrm{r}}^{\prime}}$.

The coordinates in $R 3$ can be derived from the coordinates in $R 2$ from

$$
\left(\begin{array}{l}
x^{\prime} \\
y^{\prime}
\end{array}\right)_{R 3}=\left(\begin{array}{cc}
\cos (\gamma) & \sin (\gamma) \\
-\sin (\gamma) & \cos (\gamma))
\end{array}\right)_{R 3}\left(\begin{array}{l}
x \\
y
\end{array}\right)_{R 2}-\left(\begin{array}{c}
r_{e} \\
0
\end{array}\right)_{R 3},
$$

where $\gamma$ is the rotation angle between the two systems (Fig. 4). Eq. (9) thus becomes

$$
\begin{aligned}
& 2\left(x_{t} x_{\mathrm{r}}-y_{t} y_{\mathrm{r}}\right) \sin (\gamma) \cos (\gamma) \\
& -\left(x_{t} y_{\mathrm{r}}+y_{t} x_{\mathrm{r}}\right)\left(\cos ^{2}(\gamma)-\sin ^{2}(\gamma)\right) \\
& -r_{E}\left(x_{t}+x_{\mathrm{r}}\right) \sin (\gamma)+r_{e}\left(y_{t}+y_{\mathrm{r}}\right) \cos (\gamma)=0
\end{aligned}
$$

Following (Helm, 2008), we proceed to the substitution $t=\tan \left(\frac{\gamma}{2}\right)$, and Eq. (11) becomes

$$
\begin{array}{r}
2\left(x_{t} x_{\mathrm{r}}-y_{t} y_{\mathrm{r}}\right) \frac{2 t}{1+t^{2}} \frac{1-t^{2}}{1+t^{2}}-x_{t} y_{\mathrm{r}}\left(\left(\frac{1-t^{2}}{1+t^{2}}\right)^{2}\right. \\
\left.-\left(\frac{2 t}{1+t^{2}}\right)^{2}\right)-r_{E} \frac{2 t}{1+t^{2}}\left(x_{t}+x_{\mathrm{r}}\right) \\
+r_{E} \frac{1-t^{2}}{1+t^{2}}\left(y_{t}+y_{\mathrm{r}}\right)=0 .
\end{array}
$$

This finally becomes

$c_{4} t^{4}+c_{3} t^{3}+c_{2} t^{2}+c 1_{t}^{1}+c_{0}=0$,

with

$$
\begin{aligned}
& c_{0}=\left(x_{t} y_{\mathrm{r}}+y_{t} x_{\mathrm{r}}\right)-r_{E}\left(y_{t}+y_{\mathrm{r}}\right) \\
& c_{1}=-4\left(x_{t} x_{\mathrm{r}}-y_{t} y_{\mathrm{r}}\right)+2 r_{E}\left(x_{t}+x_{\mathrm{r}}\right) \\
& c_{2}=-6\left(x_{t} y_{\mathrm{r}}+y_{\mathrm{r}} x_{\mathrm{r}}\right) \\
& c_{3}=4\left(x_{t} x_{\mathrm{r}}-y_{t} y_{\mathrm{r}}\right)+2 r_{E}\left(x_{t}+x_{\mathrm{r}}\right) \\
& c_{4}=\left(x_{t} y_{\mathrm{r}}+y_{t} x_{\mathrm{r}}\right)+r_{E}\left(y_{t}+y_{\mathrm{r}}\right) .
\end{aligned}
$$

Equation (13) is solved to determine the roots of this polynomial using an iterative scheme based on the Newton method (Nocedal et al., 2006). 


\subsection{Ellipsoid reflection approximation}

We consider an ellipsoid corresponding to the WGS84 one extended such that the ellipsoid height of the receiver is equal to the receiver height above the reflecting surface. In other words, the WGS84 ellipsoid is expanded until its surface coincides with the reflecting surface, at the nadir of the receiver (surface base point). The Cartesian coordinates of this surface base point must remain unchanged when computed either from the original geodetic coordinates $(\lambda, \phi, h)_{\text {WGS } 84}$ and the ellipsoid constant $(a, b)_{\text {WGS84 }}$ on the one hand, or their modified values $\left(\lambda^{\prime}, \phi^{\prime}, h^{\prime}, a^{\prime}, b^{\prime}\right)$ on the other hand, where $\lambda=\lambda^{\prime}$ is the longitude, $\phi=\phi^{\prime}$ is the latitude, and $h^{\prime}=0$. The ellipsoid thus remains geocentric, and its axes are scaled as follows:

$a^{\prime}=\sqrt{a^{2}+h^{2}+h c+\frac{a^{2} h}{c}}=a \sqrt{1+\frac{h^{2}}{a^{2}}+\frac{h c}{a^{2}}+\frac{h}{c}}$
$b^{\prime}=\sqrt{b^{2}+h^{2}+h c+\frac{b^{2} h}{c}}=b \cdot \sqrt{1+\frac{h^{2}}{b^{2}}+\frac{h c}{b^{2}}+\frac{h}{c}}$,

where $c=\sqrt{a^{2} \cos (\phi)^{2}+b^{2} \sin (\phi)^{2}}$

This ellipsoidal extension is only done once as long as the receiver position remains unchanged with respect to the reflecting surface; it is redone if the reflecting surface changes (e.g., tidal waters), but is not done with changes in the satellite direction.

We define the two normalized anti-incident $\boldsymbol{r}_{t}$ and scattering $\boldsymbol{r}_{s}$ vectors. When the Snell-Descartes law is verified, the sum of these two vectors (bisecting vector $\boldsymbol{d} \boldsymbol{r}$ ) coincides with the local vertical. The determination of the location of the reflection point is based on the iterative process proposed earlier by Gleason et al. (2009), and enhanced with a dichotomy process. Let us consider three points on the ellipsoid:

- $S 1$, the projection of the receiver on the ellipsoid.

- S3, the projection of the transmitter on the ellipsoid.

- $S 2$, the projection of the middle of [ $S 1 S 3]$ on the ellipsoid.

We calculate $\boldsymbol{d} \boldsymbol{r}$, the correction in direction, considering the location of each of the three points:

$\boldsymbol{d r}(t)=\frac{\boldsymbol{r}_{s}(t)-\boldsymbol{r}_{\mathrm{r}}(t)}{\left\|\boldsymbol{r}_{s}(t)-\boldsymbol{r}_{\mathrm{r}}(t)\right\|}+\frac{\boldsymbol{r}_{s}(t)-\boldsymbol{r}_{t}(t)}{\left\|\boldsymbol{r}_{s}(t)-\boldsymbol{r}_{t}(t)\right\|}$.

We then consider the direction of the correction $\boldsymbol{d r}$. If the correction is in the satellite direction, the sign is considered positive, and negative if the correction is in the receiver direction. If the signs of $d r_{S 1}$ and $d r_{S 2}$ are different, it means that the specular reflection point is located between $S 1$ and $S 2$. We thus consider a new iteration with $S 1=S 1, S 3=S 2$ and $S 2$ the projection on the ellipsoid of the middle of the new $S 1$ and $S 3$ points. We thus eliminate the part between the initial

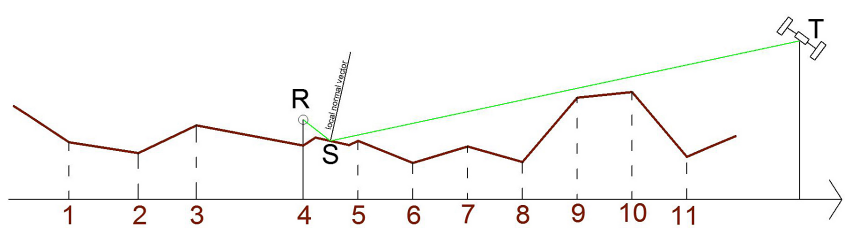

Figure 5. Determination of the specular reflection point integrating a DEM. $S$ : specular reflection point position. $R$ : receiver position. $T$ : transmitter/satellite position. A dichotomous process is applied for each topographic segment of the DEM to find if there is a point where the bisecting angle (equal to the sum of the anti-incident and scattering vectors) is colinear with the local normal vector.

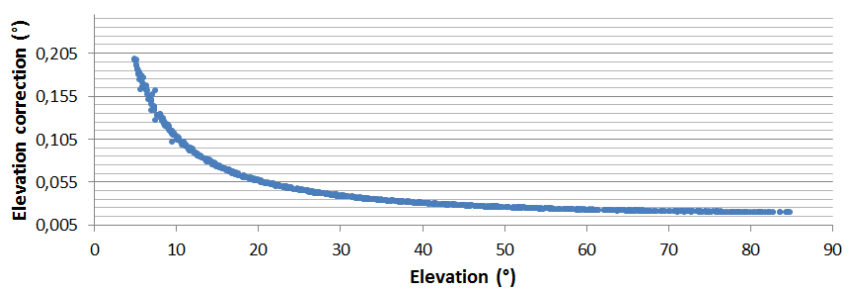

Figure 6. Effect of the neutral atmosphere on the elevation angle. An exponential correction must be made for satellites with low elevation angles.

$S 2$ and $S 3$ points, else if the signs of $d r_{S 2}$ and $d r_{S 3}$ are different, we consider a new iteration with $S 1=S 2$ and $S 3=S 3$ (and $S 2$ the projection on the ellipsoid of the middle of the new $S 1$ and $S 3$ points). The iterative process stops when the difference between incident and reflected angle (with respect to the local vertical) is close to zero with a fixed tolerance of $10^{-7 \circ}$.

\subsection{Ellipsoid reflection approximation combined with a DEM}

The first two approaches presented above are well adapted in the case of an isolated receiver located on the top of a lighthouse, for instance. In most of the cases, the receiver is located on a cliff, a sand dune, or a building overhanging the sea surface or a lake. It can however be really appropriate and necessary to incorporate a digital elevation model (DEM) into the simulations, in order not only to take the mask effects (e.g., a mountain occulting a GNSS satellite) into account, but also to get more accurate and realistic positions of specular reflection points. The method we propose here consists of three steps detailed later in Sects. 3.4.1, 3.4.2 and 3.4.3.

1. A "visibility" determination approach to determine if the receiver is in sight of each GNSS satellite.

2. A determination of the specular reflection point position. 
Table 1. Cross-validation between ellipsoid approximation and DEM integration.

\begin{tabular}{llrrr}
\hline & & \multicolumn{3}{c}{ Receiver height $(\mathrm{m})$} \\
\cline { 3 - 5 } & & 5 & 50 & 300 \\
\hline Distance to the specular reflection point & Mean & 12 & 122 & 729 \\
with respect to the receiver: arc length (m) & Maximum & 56 & 557 & 3349 \\
Position differences (m) & Mean & $0 / 0$ & $0.002 / 0$ & $0.01 / 0$ \\
(planimetric/altimetric) & Maximum & $0 / 0$ & $0.04 / 0$ & $0.2 / 0$ \\
\hline
\end{tabular}

3. A "visibility" determination approach to determine if the determined specular point is in sight from both receiver and satellite.

It is important to keep in mind that a DEM gives altitudes above a reference geoid. For consistency purposes, the positions of the receiver, the transmitter, and the DEM grid points all have to be in the same reference system, so it is absolutely mandatory to convert the EGM96 altitudes from the SRTM DEM into WGS84 ellipsoidal heights by adding the geoid undulation interpolated from EGM96.

\subsubsection{Visibility of the GNSS satellite from the receiver}

This algorithm aims to determine the presence of a mask between the receiver and the satellite. The visibility of the satellite and the receiver, both from the specular point, will be checked once the potential specular point position has been found.

Let $R, S$, and $T$ be the locations of the receiver, the specular point and the satellite/transmitter on the ellipsoid. We interpolate the ellipsoidal heights along the path $[T S R]$ with a step equal to the DEM resolution, with a bivariate cubic or bilinear interpolation. Cubic interpolation is used when the gradient is big, linear interpolation otherwise. Tests show millimetric differences between cubic and linear interpolation for flat zones but can reach $1 \mathrm{~m}$ for mountainous areas. We thus obtain a topographic profile from $R$ to $T$. For each segment of this topographic profile, we check if it intersects the path $[T R]$. If it does, it means that the satellite is not visible from the receiver. If not, we check the next topographic segment, until reaching the end of the path (i.e., $T$ ).

\subsubsection{Position of the specular point}

Once the satellite visibility from the receiver is confirmed, the next step consists in determining the location of the specular reflection point $S$ along the broken line defined as in Sect. 3.4.1. In order to simplify the process, we only consider the specular points located in the plane formed by the satellite, the receiver and the center of the Earth. The algorithm is similar to the one used for the ellipsoid approximation, and is based on a dichotomous iterative process.
The segments formed by the points of the 2-D DEM (see Fig. 5) are all considered susceptible to contain a specular reflection point. For each of this segment the sign of the correction to apply at each of the two extremities of the segment is checked following the same principle that for the ellipsoid approximation (see Sect. 3.3), but with a local vertical component defined as the normal of the considered segment. If the signs are equal, no reflection is possible on this segment. Otherwise, we apply the dichotomous iterative method presented in Sect. 3.3 until convergence with respect to the tolerance parameter (fixed to $10^{-7 \circ}$ ).

\subsubsection{Visibility of the determined specular reflection point from the satellite and the receiver}

Once the position of the specular reflection point has been determined, we check if it is visible from the satellite and the receiver thanks to the algorithm presented in Sect. 3.4.1.

\subsection{Corrections of the angular refraction due to the troposphere}

Our goal is to determine the location of the reflection point. Only the angular refraction will be considered. The reflected minus direct range is left as future work. In order to correct the anisotropy of the propagation of radio waves used by the GNSS satellites, we use AMF calculated from the 3-hourly delayed cut-off in model levels computed by the ECMWF (European Centre for Medium-Range Weather Forecasts). AMF tropospheric corrections were computed following Gegout et al. (2011) and provided by GRGS for this study. Given the geometric specificities of the specular reflection point, two paths have to be checked for propagation error: the first one from the satellite to the surface, and the second one from the surface to the receiver. The main steps of the process are the following:

- we consider the position of the specular reflection point without any correction of the angular refraction;

- we calculate the corrections to apply to this specular point knowing the incident and reflecting angles corresponding to the considered reflection point. We thus obtain a corrected incident angle. Figure 6 shows the correction to apply as a function of the elevation angle; 
- from the corrected incident angle, a corrected position of the specular point is calculated, making the reflecting angle equal to the corrected incident angle;

- with the new position of the specular point, and to reach a better accuracy of the point position, a second iteration is performed by computing the corrections to apply to this new incident angle.

\subsubsection{Correction of the satellite-surface path}

First and foremost, the parallax problem for the wave emitted by a known GNSS satellite is solved. At first sight, the position of the specular reflection point calculated without any correction of the angular refraction is considered, given by the algorithm approximating the Earth's shape as a sphere given in Sect. 3.2. We use here AMF calculated from the projection of the receiver on the surface, considering that the AMF planimetric variations are negligible for ground-based observations (i.e., we consider that we can use the same AMF for every specular reflection points, which is valid only if the specular reflection points are less than few tens of $\mathrm{km}$ from the receiver and that the specular points lie on an equalheight surface). We thus obtain the corrected incident angle of the incident wave. Considering the law of Snell and Descartes, the reflecting angle must be equal to the corrected incident angle, for the specular reflection point position.

\subsubsection{Correction of the surface-receiver path}

The aim here is to adjust the surface-receiver path to accommodate the consequences of angular refraction. With the corrected reflection angle, we can deduce the corrected geometric distance between the reflection point and the receiver, this time using AMF calculated from the receiver, assuming that the AMF altimetric variations are non-negligible (i.e., the part of the troposphere corresponding to the receiver height will have a non-negligible impact on the AMF). Considering the corrected geometric distance between the reflection point and the receiver, the corrected position of the reflection point is obviously determined. It is indeed obtained as the intersection of a circle whose radius is equal to the correct geometric distance, with the surface of the Earth assimilated as a sphere, an ellipsoid, or with a DEM, depending on which approximation of the Earth is taken into account.

The whole process is iterated a second time to reach a better accuracy of the reflection point location. In fact, the first corrections were not perfectly exact, since they were computed from an initially false reflection point location, and the second iteration brings the point closer to the true location. More iterations are useless (corrections to apply are not significant). Figure 6 shows an example of elevation corrections to apply as functions of the satellite elevations. This figure has been computed from simulations done on a receiver placed on the Lake Geneva shore $\left(46^{\circ} 24^{\prime} 30^{\prime \prime} \mathrm{N}, 6^{\circ} 43^{\prime} 6^{\prime \prime} \mathrm{E}\right.$; $471 \mathrm{~m}$ ); see Sect. 4.1.

\subsection{Footprint size of the reflected signal}

The power of the received signal is mostly due to coherent reflection, and most of the scattering comes from the first Fresnel zone (Beckmann and Spizzichino, 1987). The first Fresnel zone can be described as an ellipse of a semi-minor axis $\left(r_{a}\right)$ and a semi-major axis $\left(r_{b}\right)$ equal to (Larson and Nievinski, 2013)

$r_{b}=\sqrt{\frac{\lambda h}{\sin \left(\epsilon^{\prime}\right)}+\left(\frac{\lambda}{2 \sin \left(\epsilon^{\prime}\right)}\right)^{2}}$

$r_{a}=\frac{b}{\sin \left(\epsilon^{\prime}\right)}$,

with $\lambda$ the wavelength (m), $h$ the receiver height (m) and $\epsilon^{\prime}$ the satellite elevation seen from the specular reflection point (rad) (i.e., corresponding to the reflection angle).

\section{Simulator performance and results}

\subsection{Simulation case studies}

Simulations and tests of parameters have been performed on two main sites:

- the Cordouan lighthouse ( $\left.45^{\circ} 35^{\prime} 11^{\prime \prime} \mathrm{N} ; 1^{\circ} 10^{\prime} 24^{\prime \prime} \mathrm{W}\right)$, in the Gironde estuary, France. This lighthouse is about $60 \mathrm{~m}$ high, and it is surrounded by the sea.

- the shore of Lake Geneva $\left(46^{\circ} 24^{\prime} 30^{\prime \prime} \mathrm{N} ; 6^{\circ} 43^{\prime} 6^{\prime \prime} \mathrm{E}\right)$. This site is hidden by mountains in the south (orthometric altitude of up to $2000 \mathrm{~m}$ ), and overlooks the lake in the north (orthometric altitude of $370 \mathrm{~m}$ ).

For both sites, precise GPS and GLONASS ephemeris at a 15 min time sampling come from IGS standard products (known as "SP3 orbit").

\subsection{Validation of the surface models}

Simulations were performed in the case of the Lake Geneva shore, for a $24 \mathrm{~h}$ experiment, on 4 October 2012.

\subsubsection{Cross-validation between sphere and ellipsoid approximations}

Local sphere and ellipsoid approximation algorithms have been compared by putting the ellipsoid semi- major and minor axis equal to the sphere radius. Planimetric and altimetric differences between both are below $6 \times 10^{-5} \mathrm{~m}$ for a receiver height above reflecting surface between 5 and $300 \mathrm{~m}$ and are then negligible. The two algorithms we compare are completely different: the first is analytical and the second is based on a iterative scheme and both results are very similar, which confirms their validity. 


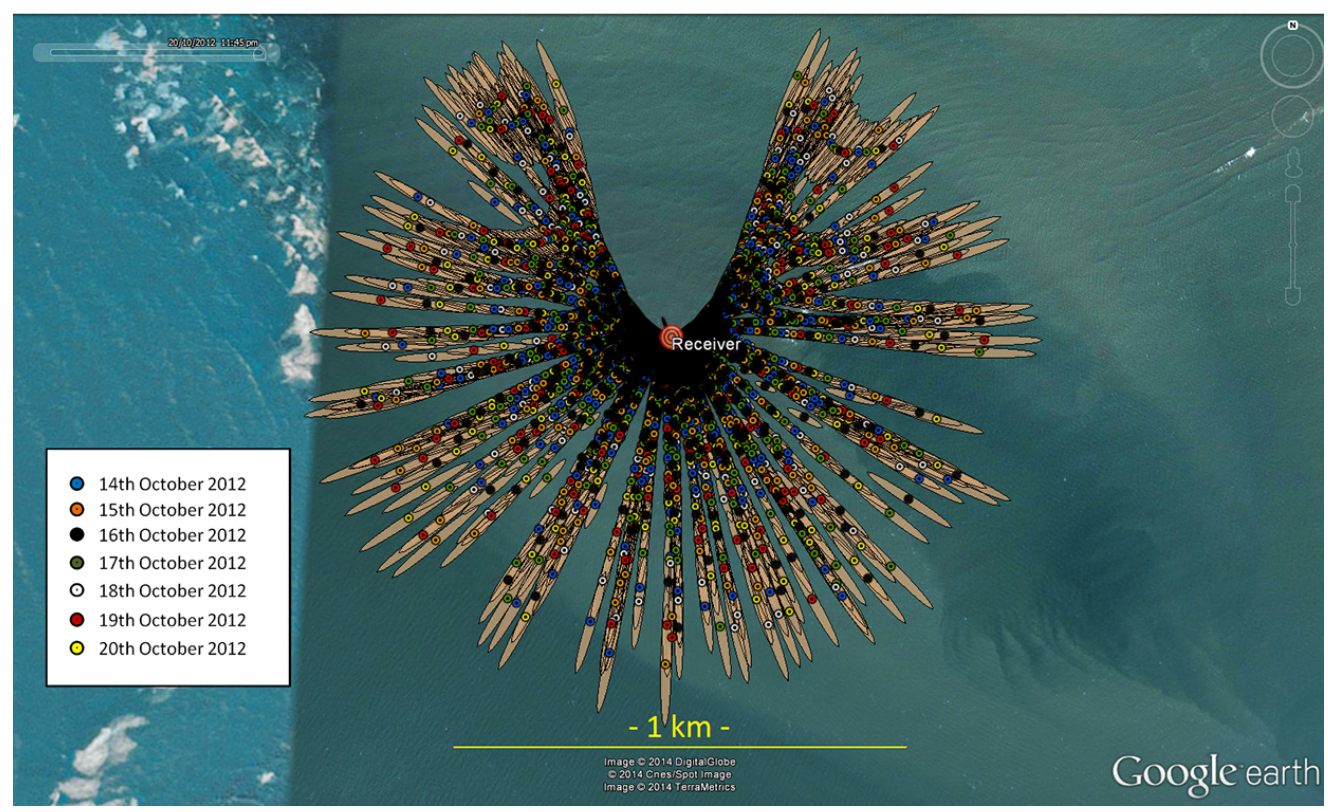

Figure 7. Positions of the specular reflection points and first Fresnel zones for one week of simulation on the Cordouan lighthouse with a 15 min sampling rate (i.e., satellite positions actualized every $15 \mathrm{~min}$ ). Only GPS satellites with elevation angles greater than $5^{\circ}$ have been considered. Note the gap in the northerly direction.

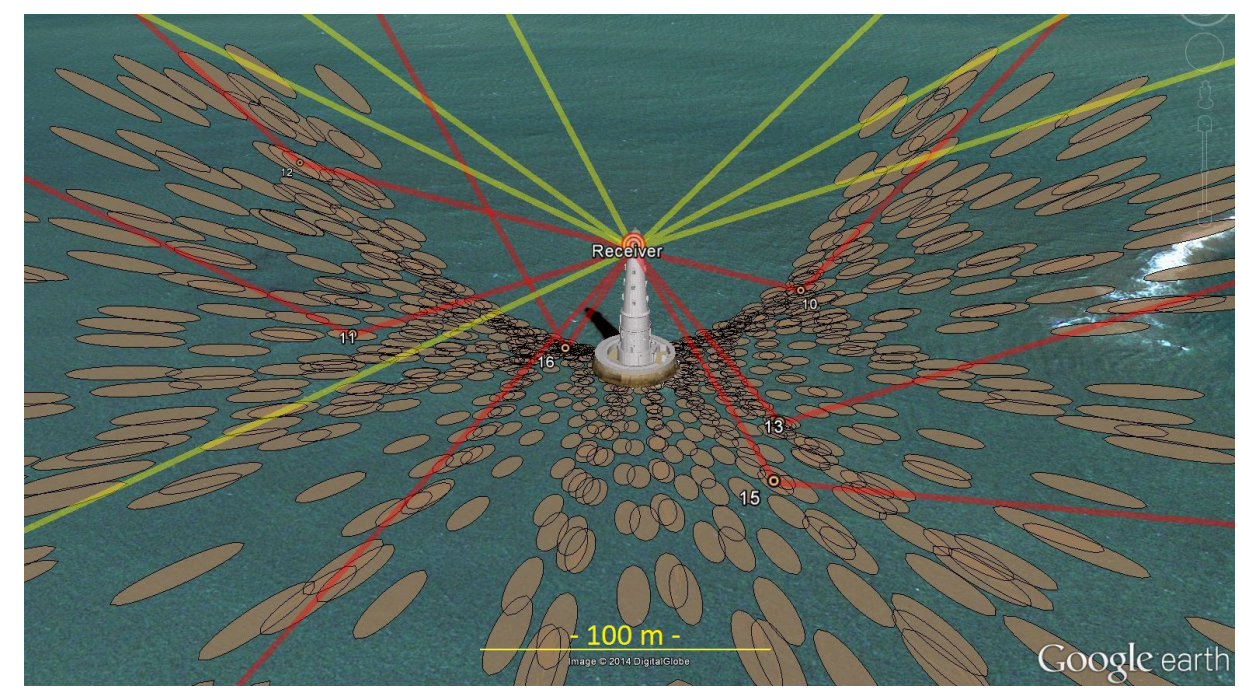

Figure 8. First Fresnel zones and some direct and reflected waves displaying the $24 \mathrm{~h}$ Cordouan lighthouse simulation with the GPS constellation.

\subsubsection{Cross-validation between ellipsoid approximation and DEM integration}

The algorithm integrating a DEM has been compared to the ellipsoid approximation algorithm by using a flat DEM as input (i.e., a DEM with orthometric altitude equal to the geoid undulation). Results for satellite elevation angles above $5^{\circ}$ are presented in Table 1.
As we can see in Table 1, planimetric and altimetric mean differences are subcentimetric for a 5 and $50 \mathrm{~m}$ receiver height and centimetric for a $300 \mathrm{~m}$ receiver height. However, some punctual planimetric differences reach $20 \mathrm{~cm}$ in the worst conditions (reflection occurring at $3449 \mathrm{~m}$ from the receiver corresponding to a satellite with a low elevation angle), which can be explained with the chosen tolerance parameters but mainly because due to the DEM resolution, the algorithm taking a DEM into account approximating the ellipsoid as a broken straight line, causing inaccuracies. For a 
a)

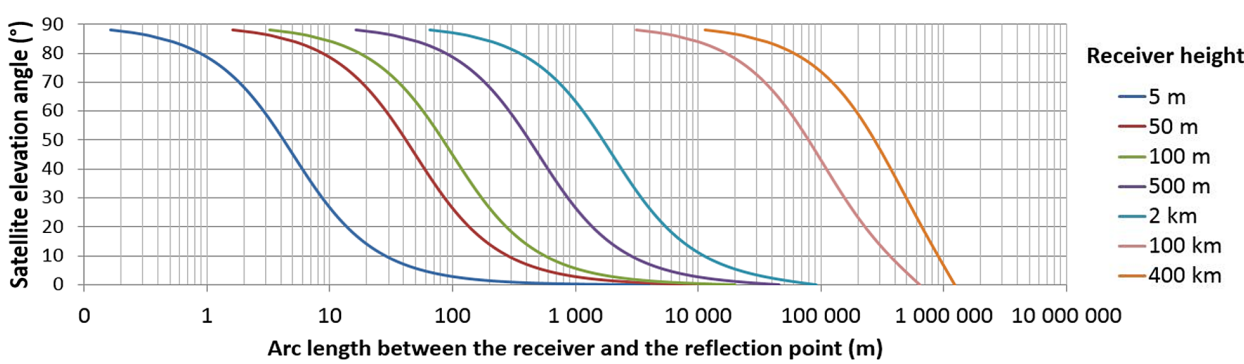

b)

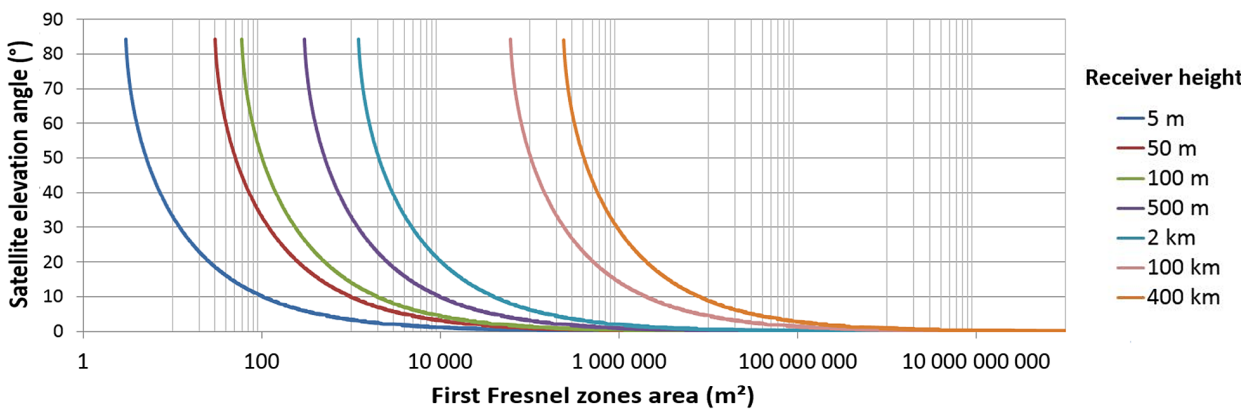

Figure 9. Variation of the distance between the receiver and the specular reflection point (a) and the first Fresnel zone area (b) as a function of the satellite elevation angle, for different receiver heights.

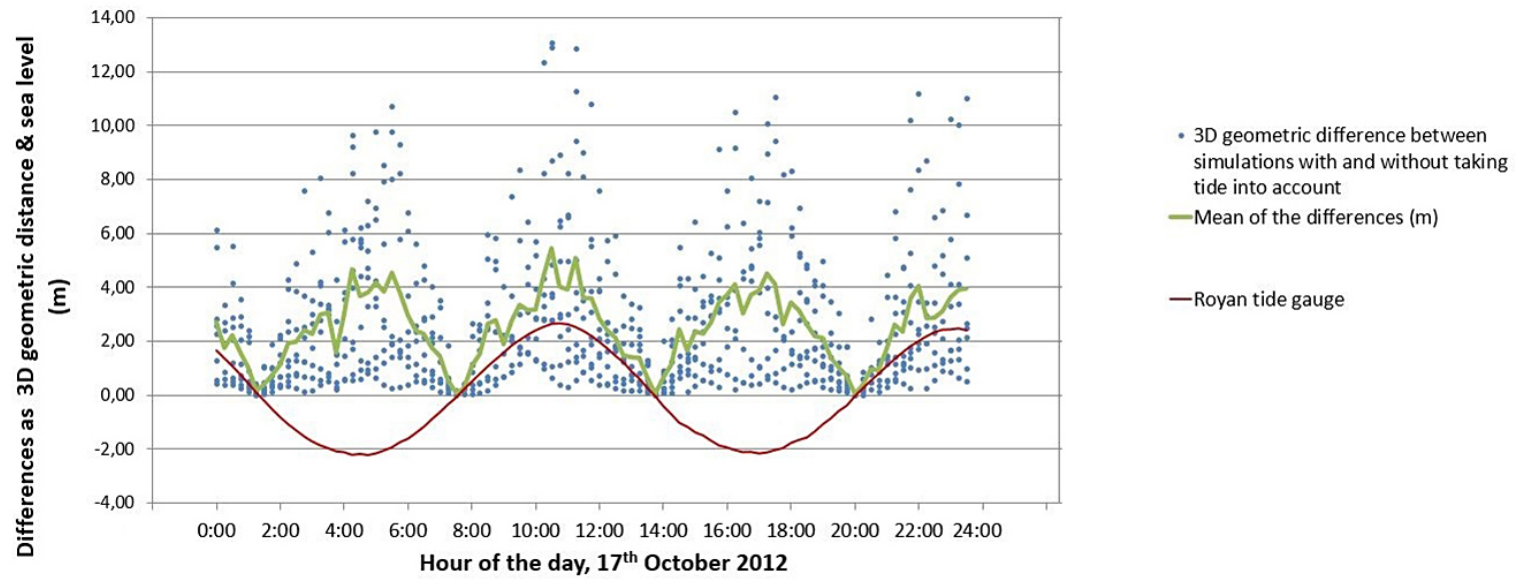

Figure 10. Assessment of the tidal influence. The impact of the tide on the size of the reflecting area is non-negligible (decametric 3-D differences), and it is worth noticing that the gaps would have been even bigger by integrating satellites with low elevation angles. Note also that the periodic variations of the 3-D differences are only linked to the tide, since the mean of the satellite elevation angles does not show periodic variations during the day of the simulation $\left(43.3 \pm 3.5^{\circ}\right.$ over the period).

$50 \mathrm{~m}$ receiver height, planimetric differences are below $4 \mathrm{~cm}$ (reflections occurring until $557 \mathrm{~m}$ from the receiver). With regards to the altimetric differences, even for reflections occurring far from the receiver, the differences are negligible (submillimetric).

\subsection{Results}

\subsubsection{Cordouan lighthouse}

\section{Outputs}

Examples of outputs for simulations in the case of the Cordouan lighthouse are presented in Figs. 7 and 8. These 
a)

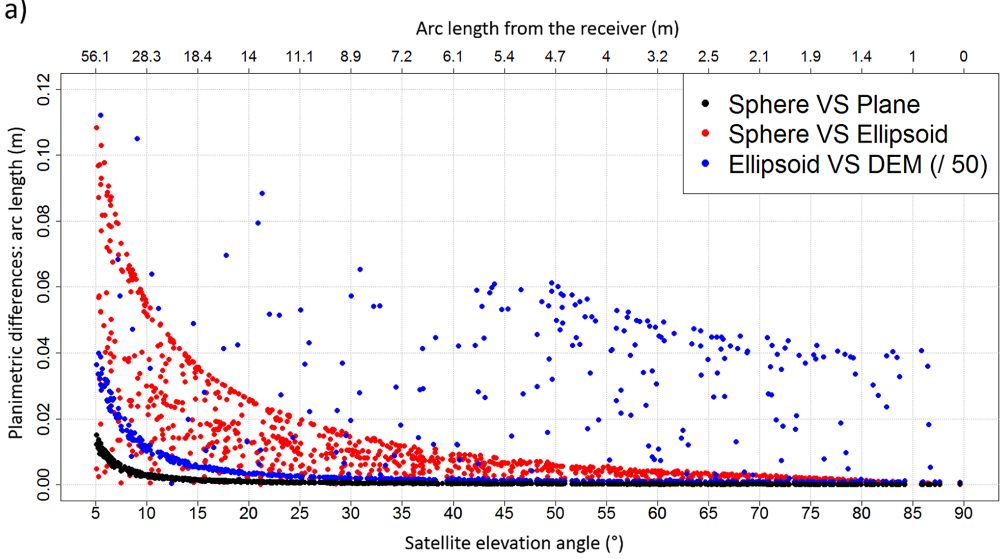

b)

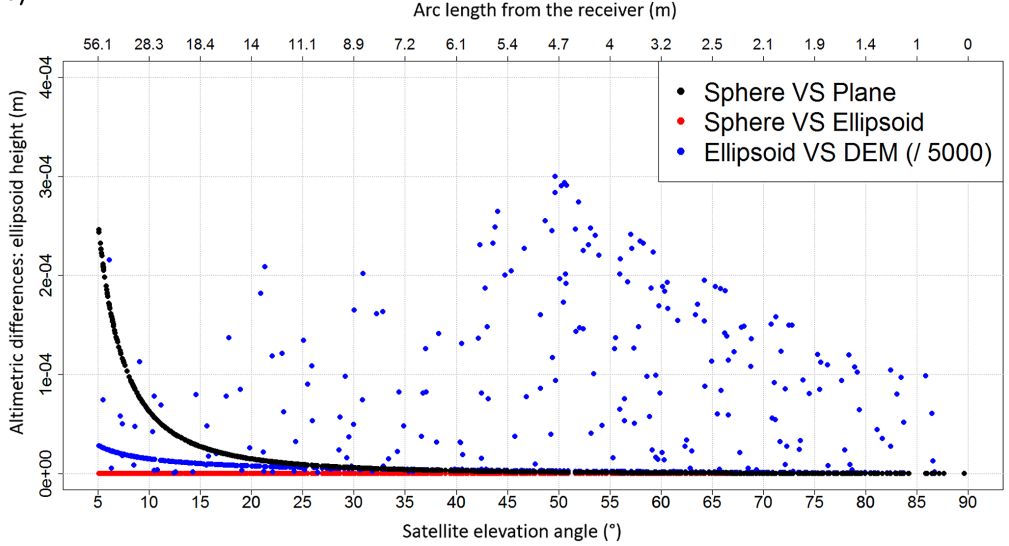

Figure 11. Planimetric and altimetric differences between the specular reflection points obtained with the different algorithms. Receiver height above the reflecting surface: $5 \mathrm{~m}$. (a) Planimetric differences as arc length (m). (b) Altimetric differences as ellipsoid height (m). Note the dispersion within results for a fixed elevation angle, which is a consequence of the azimuth variability in the ellipsoidal radius of curvature.

simulations were performed considering the sphere approximation algorithm and a 15 min time step.

Figure 9a shows the variation of the distance between reflected points and the receiver, as a function of the satellite elevation angle, and for several receiver heights above the reflecting surface, and Fig. 9b shows the variation of the area of the first Fresnel surface. Such figures have been produced by performing simulations on the Cordouan lighthouse and varying the receiver height above the reflecting surface. The map of the reflected points obtained for a high receiver height above the reflecting surface will in fact be the same as the one obtained for a smaller receiver height, but more stretched. Henceforth, the higher the receiver height, the bigger the "measurable" area, but the less dense the ground coverage of the data (less reflection points per surface unit).

\section{Case study: the influence of tides}

As an illustration of a possible application of the simulator, tide influence on the position of the specular reflection points was assessed. Simulations at the Cordouan lighthouse were achieved by integrating the ocean tide from the tide gauge in Royan, and by time-varying the receiver height above the sea surface in order to simulate the tide. The vertical visibility mask was set to $10-90^{\circ}$, in order to avoid the weaker accuracy of determination of the specular reflection point positions for satellites with low elevation angles, as highlighted in Sect. 4.3.2. By comparing the results with simulations made with a fixed-receiver height of $60 \mathrm{~m}$ above the sea surface, it appears that the 3-D offsets reach values higher than $12 \mathrm{~m}$ for the maximum tide values $(<3 \mathrm{~m})$ (Fig. 10). We can expect even higher discrepancies by taking into account satellites whose elevation angles would be lower than $10^{\circ}$.

\subsubsection{Lake Geneva}

Three sets of simulation have been performed in the case of the Lake Geneva shore, for a $24 \mathrm{~h}$ experiment, on 4 October 2012: 
Table 2. Maximum differences between the positions of the specular reflection points obtained with the different algorithms and for different receiver heights above the reflecting surface. For each cell of this table, the first number is the result obtained with the minimum satellite elevation angle set to $5^{\circ}$, and the second number is the result obtained with the minimum satellite elevation angle set to $10^{\circ}$.

\begin{tabular}{llccc}
\hline $\begin{array}{l}\text { Receiver } \\
\text { height }(\mathrm{m})\end{array}$ & $\begin{array}{l}\text { Differences } \\
(\mathrm{m})\end{array}$ & Sphere vs. Plane & sphere vs. ellipsoid & Ellipsoid vs. DEM \\
\hline \multirow{3}{*}{5} & Arc length & $0.015 / 0.003$ & $0.108 / 0.054$ & $14.594 / 4.417$ \\
& Ellipsoid height & $0 / 0$ & $0 / 0$ & $1.500 / 1.500$ \\
& 3-D geometric distance & $0.011 / 0.002$ & $0.084 / 0.044$ & $10.261 / 3.383$ \\
\hline \multirow{3}{*}{50} & Arc length & $1.163 / 0.142$ & $1.081 / 0.536$ & $1226.606 / 42.982$ \\
& Ellipsoid height & $0.025 / 0.006$ & $0 / 0$ & $84.363 / 15.002$ \\
& 3-D geometric distance & $0.823 / 0.107$ & $0.837 / 0.440$ & $1235.834 / 43.755$ \\
\hline \multirow{3}{*}{300} & Arc length & $41.127 / 5.043$ & $6.438 / 3.215$ & $5429.975 / 5429.975$ \\
& Ellipsoid height & $0.885 / 0.222$ & $0.001 / 0$ & $897.785 / 897.785$ \\
& 3-D geometric distance & $29.092 / 3.769$ & $4.994 / 2.634$ & $5461.230 / 5461.230$ \\
\hline
\end{tabular}

a)

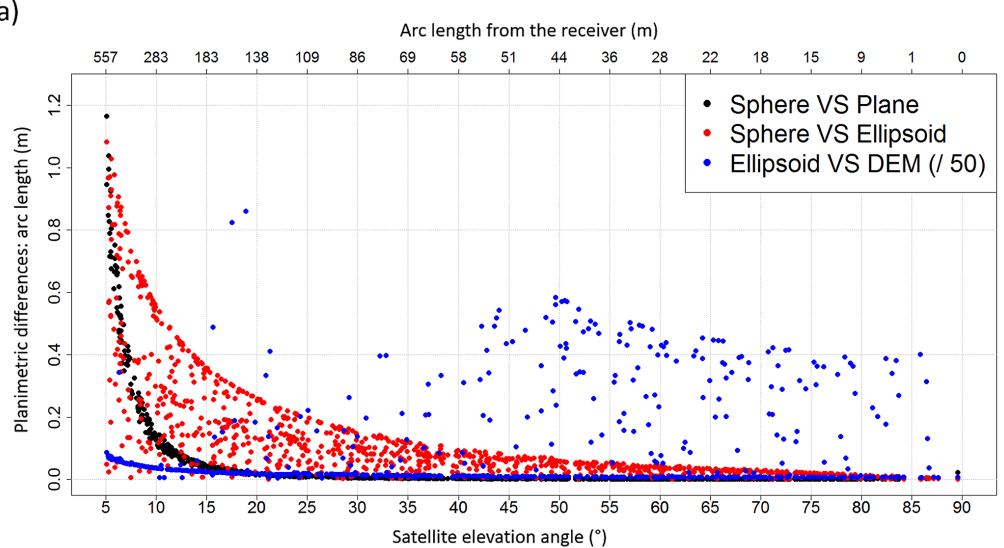

b)

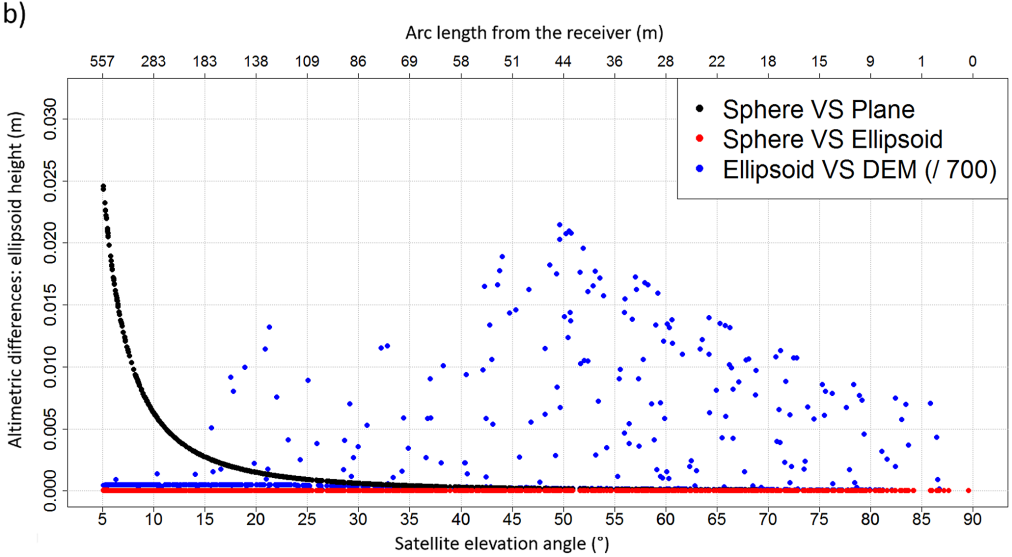

Figure 12. Planimetric and altimetric differences between the specular reflection points obtained with the different algorithms. Receiver height above the reflecting surface: $50 \mathrm{~m}$. (a) Planimetric differences as arc length (m). (b) Altimetric differences as ellipsoid height (m). Note the dispersion within results for a fixed elevation angle, which is a consequence of the azimuth variability in the ellipsoidal radius of curvature. 
a)

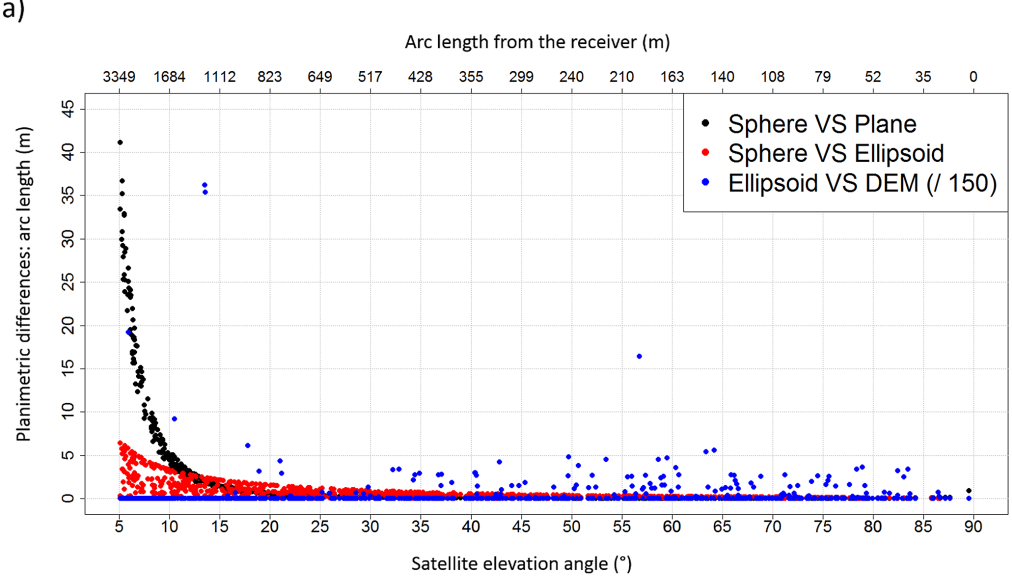

b)

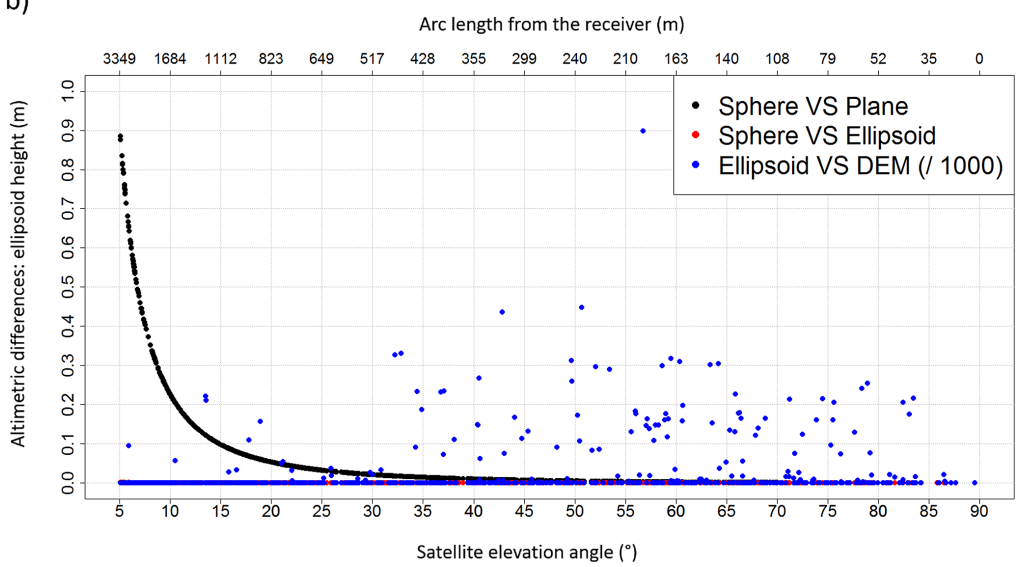

Figure 13. Planimetric and altimetric differences between the specular reflection points obtained with the different algorithms. Receiver height above the reflecting surface: $300 \mathrm{~m}$. (a) Planimetric differences as arc length (m). (b) Altimetric differences as ellipsoid height (m). Note the dispersion within results for a fixed elevation angle, which is a consequence of the azimuth variability in the ellipsoidal radius of curvature.

- the first configuration, considering a receiver height of $5 \mathrm{~m}$ above lake level,

- the second configuration, considering a receiver height of $50 \mathrm{~m}$ above lake level,

- the third configuration, considering a receiver height of $300 \mathrm{~m}$ above lake level, as for an airborne experiment (e.g., a hovering helicopter).

Each series has been computed using the four algorithms of determination of the reflection points (local planimetric approximation, local osculating sphere approximation, ellipsoid approximation and the algorithm taking a DEM into account). Results are presented in Figs. 11 to 14 and in Table 2. They show the distances between the specular points and the receiver (arc lengths), and the differences between the positions given by each algorithm.

\section{Influence of the receiver height above the reflecting surface}

It appears that both planimetric and altimetric differences between the methods used increase with the receiver height above the reflecting surface. This is explainable by the fact that the higher the receiver is, the farther the reflection points will be from the receiver, and the bigger the impact of the Earth approximation will be. For a $5 \mathrm{~m}$ receiver height, reflection occurs up to approximately $60 \mathrm{~m}$ from the receiver, whereas for a $300 \mathrm{~m}$ receiver height, it occurs at up to $3400 \mathrm{~m}$ (6700 $\mathrm{m}$ when integrating the DEM). It means that, in the second case, reflections occur in the mountains to the south of the receiver; hence big differences between the sphere algorithm and the algorithm, taking the DEM into account. For a $5 \mathrm{~m}$ receiver height above the reflecting surface, and considering satellites with elevation angles above $5^{\circ}$, mean planimetric differences are below $1.3 \mathrm{~cm}$ between the osculating sphere and ellipsoid approximations, and below $1.3 \mathrm{~mm}$ between the sphere and plane approximations. With regards to 
a)

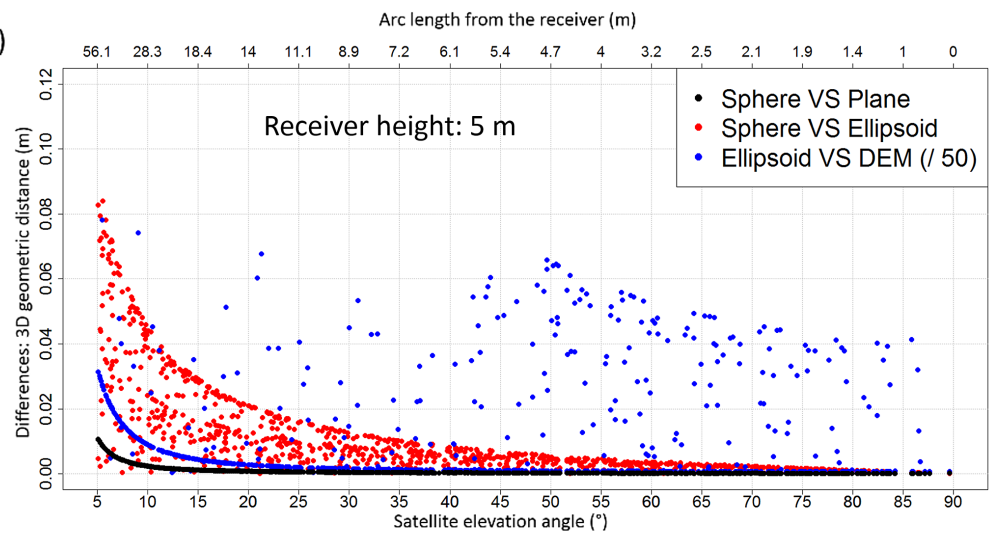

b)

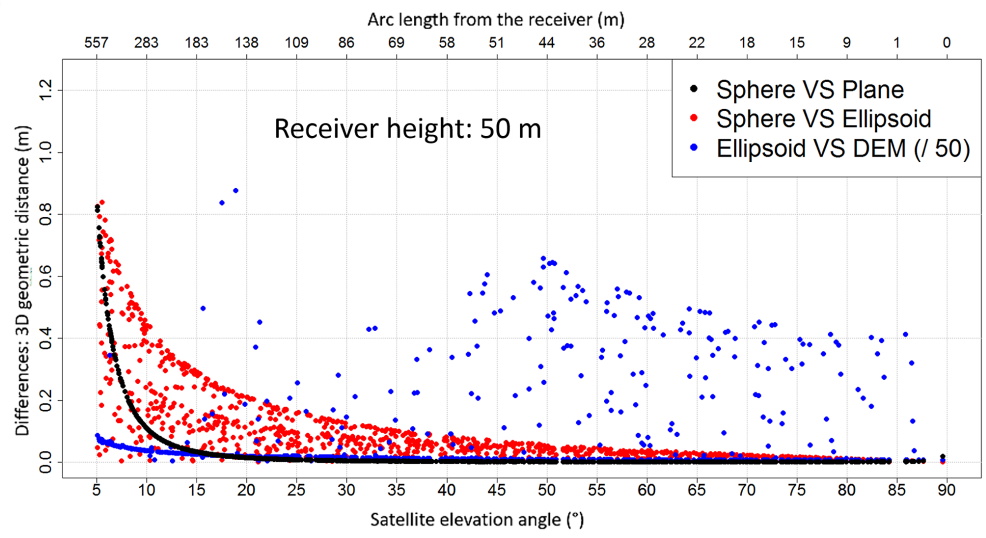

c)

Arc length from the receiver $(\mathrm{m})$

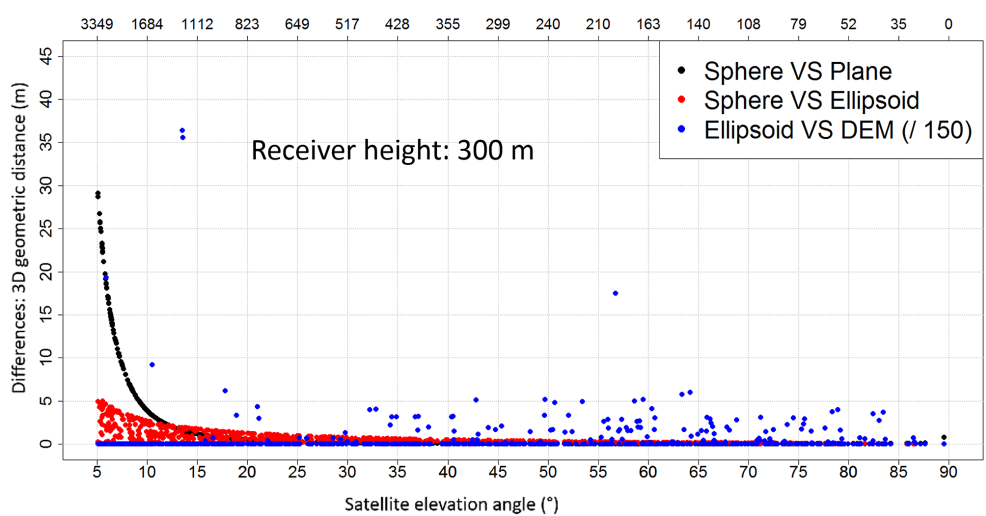

Figure 14. 3-D differences between the specular reflection points obtained with the different algorithms. Receiver heights above the reflecting surface of $5 \mathrm{~m} \mathrm{(a),} 50 \mathrm{~m} \mathrm{(b)}$ and $300 \mathrm{~m}$ (c). Note the dispersion within results for a fixed elevation angle, which is a consequence of the azimuth variability in the ellipsoidal radius of curvature.

the comparison between the plane and ellipsoid approximations, the mean planimetric differences are about $1.4 \mathrm{~cm}$. Altimetric differences are negligible for all of them.

With a $50 \mathrm{~m}$ receiver height above the reflecting surface, mean planimetric (or altimetric) differences reach $14 \mathrm{~cm}$ (or less than $1 \mathrm{~mm}$ ) between the sphere and ellipsoid approximations, $6.2 \mathrm{~cm}$ (or $2 \mathrm{~mm}$ ) between the sphere and plane approximations, and $15 \mathrm{~cm}$ (or $2 \mathrm{~mm}$ ) between the plane and ellipsoid approximations.
With a $300 \mathrm{~m}$ receiver height above the reflecting surface, mean planimetric (or altimetric) differences reach $83 \mathrm{~cm}$ (or less than $1 \mathrm{~mm}$ ) between the sphere and ellipsoid approximations, $2.19 \mathrm{~m}$ (or $8 \mathrm{~cm}$ ) between the sphere and plane approximations, and $2.35 \mathrm{~m}$ (or $8 \mathrm{~cm}$ ) between the plane and ellipsoid approximations. 


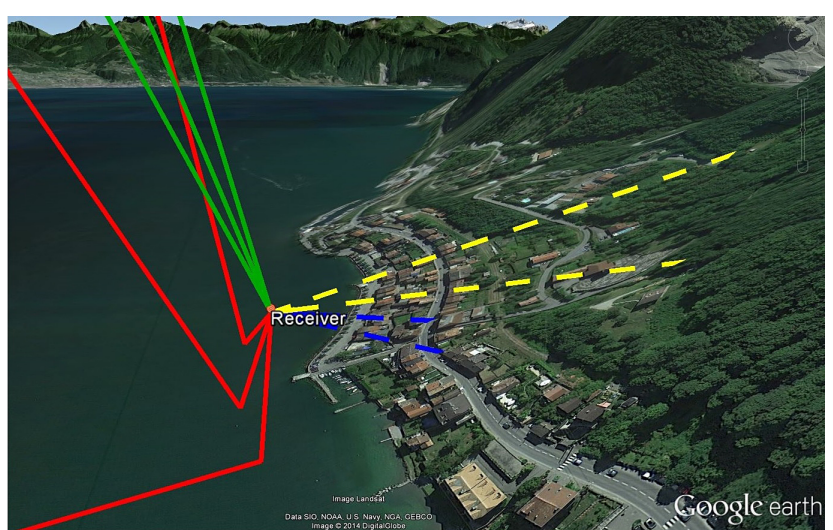

Figure 15. Influence of the topography - direct and reflected waves are displayed (topography amplified by a factor of 3). Yellow lines: direct waves, sphere approximation algorithm; green lines: direct waves, taking a DEM into account; blue lines: reflected waves, sphere approximation algorithm; red lines: reflected waves, taking a DEM into account. It can be noticed that some yellow and blue lines (direct and reflected waves, sphere approximation algorithm) go through the mountain (reflection points having been calculated inside the mountain), whereas any red or green lines (direct and reflected waves, integrating a DEM) go through it.

It is worth noticing that the sphere approximation is closer to the plane than the ellipsoid approximation when reflections occur not too far from the receiver (below $560 \mathrm{~m}$ ), and conversely if reflection occurs far from the receiver.

\section{Influence of the satellite elevation angle}

Secondly, by plotting the differences as functions of the satellite elevation angles, we can observe that the lapses between the different algorithms vary in an inversely proportional way to the satellite elevation angle (and so, proportionally to the point distance from the receiver). The lower the satellite elevation angle is, the farther the specular reflection points from the receiver, and the larger the impact of the Earth approximation is. The choice of the algorithm used to perform the simulations thus becomes really important for the farthest reflection points (i.e., for low satellite elevation angles, and high receiver heights above the reflecting surface). For example, mean planimetric differences between the local sphere and ellipsoid approximations with a $50 \mathrm{~m}$ receiver height are about $14 \mathrm{~cm}$ when considering satellites with elevation angles above $5^{\circ}$, and are about $9 \mathrm{~cm}$ when considering only satellites with elevation angles above $10^{\circ}$. With a $300 \mathrm{~m}$ receiver height above the reflecting surface, the mean planimetric difference between sphere and ellipsoid approximations is about $83 \mathrm{~cm}$ for satellites with an elevation angle above $5^{\circ}$, and $54 \mathrm{~cm}$ for a minimum elevation angle set to $10^{\circ}$.

\section{Influence of the DEM integration}

For continental surfaces, the full integration of the DEM in the simulation plays a crucial role in a good calculation of the reflection points. The integration of a DEM leads to the suppression of 245 specular reflection points out of the 905 points determined during the $24 \mathrm{~h}$ of 4 October 2012 with the sphere approximation algorithm (Fig. 15). These 245 points came from a wave emitted by a satellite hidden by a mountain located in the southern part of the area. In the northern part, any reflection point is valid when taking a DEM into account, because in that direction, the topography is flat over Lake Geneva, and so, the satellites are all visible and reflections are possible. Moreover, the point positions have been rectified while taking a DEM into account, since the other algorithms consider that reflections occur (in first approximation) in a plane around the projection of the receiver, and without integrating the problem of the presence of topography.

\section{Comparison between the different models of the Earth surface}

For a $5 \mathrm{~m}$ receiver height, and for satellite elevations greater than $10^{\circ}$, the mean planimetric difference between the ellipsoid and the sphere algorithm is equal to $1.4 \mathrm{~cm}$ whereas for a $300 \mathrm{~m}$ receiver height it is equal to $83 \mathrm{~cm}$. The approximation done by considering the Earth as a sphere, an ellipsoid or a plane does not really affect the precision of the specular reflection point determination when reflections do not occur too far from the receiver, i.e., for low receiver height and high satellite elevation. For example, if we consider that we need an uncertainty on the determination of the specular reflection position below $20 \mathrm{~cm}$, the choice of the approximation of the Earth shape will have no influence if reflections occur until $125 \mathrm{~m}$ approximately (Fig. 14b). In order to get reflections below $125 \mathrm{~m}$ from the receiver, considering satellites with elevation angle above $5^{\circ}$, the receiver height above the reflecting surface should not exceed 25-30 m (Fig. 9a), which would correspond to a first Fresnel zone area between 300 and $400 \mathrm{~m}^{2}$.

Concerning the algorithm taking the DEM into account, the differences obtained with respect to the sphere or ellipsoid algorithms are quite big even if the specular reflection point is close enough to the receiver. For instance, the mean planimetric (or altimetric) difference between the ellipsoid algorithm and the one integrating the DEM is equal to $70 \mathrm{~cm}$ (or $18 \mathrm{~cm}$ ) for a $5 \mathrm{~m}$ receiver height, and is equal to $78 \mathrm{~m}$ (or $25 \mathrm{~m}$ ) for a $300 \mathrm{~m}$ receiver height, and with a satellite elevation angle above $5^{\circ}$. It is worth noticing that these differences will greatly depend on the flatness of the considered area. 

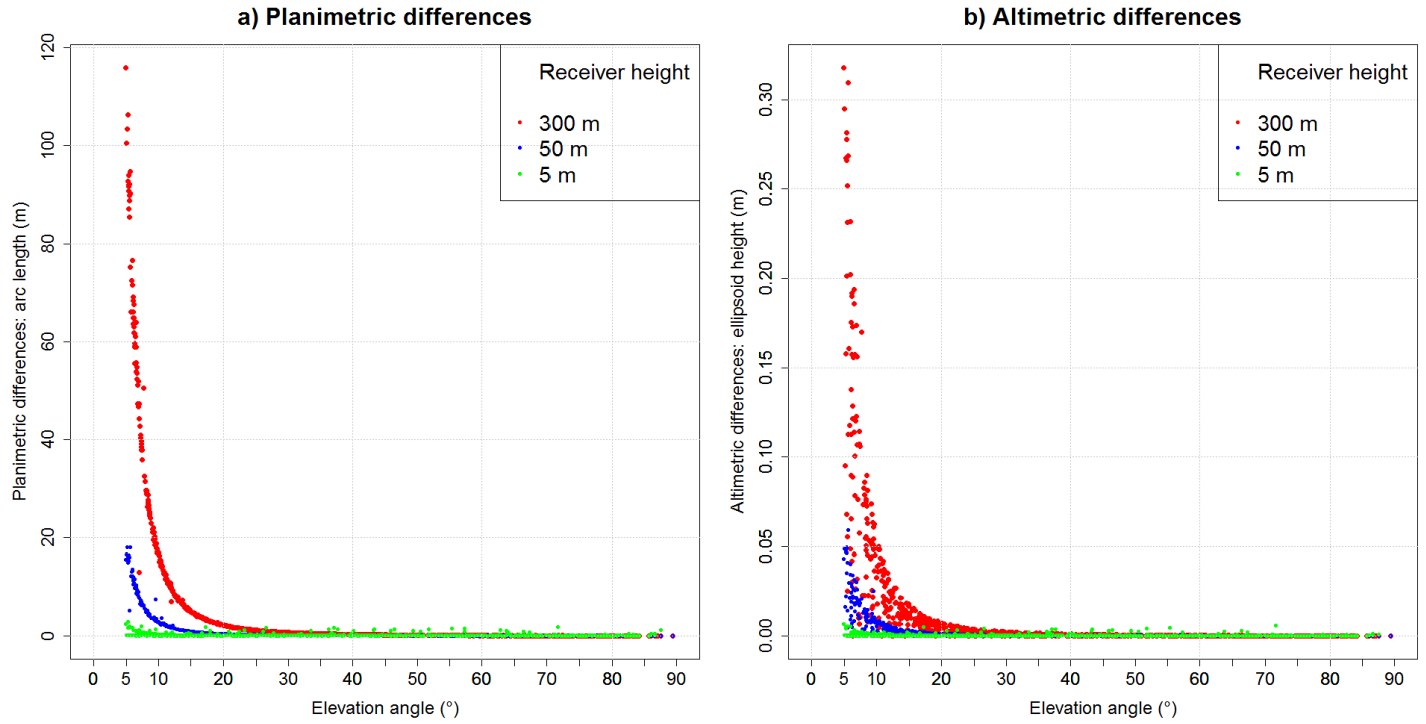

Figure 16. Angular refraction correction as a function of the satellite elevation angle and for different receiver heights above the reflecting surface. (a) Planimetric differences as arc length (m). (b) Altimetric differences as ellipsoid height (m).

\section{Angular refraction due to the troposphere}

Given the geometric configuration of the satellite, the reflection point and the receiver, the same elevation angle correction will have a different effect according to the receiver height above the reflecting surface. It turns out that considering a same satellite at a given time, the corresponding reflection point will be farther for a big receiver height above the reflecting surface than for a smaller one. Consequently, for the same elevation angle correction, the resulting correction of the reflection point position will be higher in the first case than in the second one. Figure 16 shows the differences, in terms of geometric distances, between the reflection points positions obtained with and without correcting the angular refraction and for different receiver heights. It appears that for low satellite elevation angle and high receiver height, the angular refraction has a non-negligible influence on the specular point positions $(116 \mathrm{~m}$ (or $32 \mathrm{~cm}$ ) for a $300 \mathrm{~m}$ receiver height and satellites elevation angle lower than $10^{\circ}$ ).

\section{Conclusions}

In this paper, we presented a simulator based on real GNSS satellite ephemeris, as a user-friendly tool for modeling the trajectories of GNSS electromagnetic waves that are reflected on the surface of the Earth and therefore preparing GNSS$\mathrm{R}$ campaigns more efficiently. The originality of this simulator remains mainly in the integration of a DEM and the correction of the angular refraction due to the troposphere. The results of simulations led us to a better understanding of the influence of some parameters on the reflection geometry, namely by quantifying the impact of the receiver height, but also the influence of the satellite elevations, the natural topography (DEM), and the troposphere perturbation.

The different simulations realized near quite rugged topography lead us to the following conclusions:

- the DEM integration is really important for mountainous areas: planimetric differences as arc length (or altimetric differences as ellipsoid height) can reach $5.4 \mathrm{~km}$ (or $1.0 \mathrm{~km}$ ) for a $300 \mathrm{~m}$ receiver height, considering satellite with elevation angle greater than $5^{\circ}$.

- differences between sphere and ellipsoid approximations are negligible for specular reflection points close to the receiver (closer than 50-60 m), i.e., small receiver heights and/or high satellite elevations. For instance, planimetric differences are smaller than $11 \mathrm{~cm}$ for a $5 \mathrm{~m}$ receiver height, considering satellites with elevation angles greater than $10^{\circ}$. Altimetric differences are negligible.

- sphere and plane approximations show really small differences in the vicinity of the receiver (smaller differences than between the sphere and ellipsoid approximations): maximum differences are about $1.5 \mathrm{~cm}$ (or $3 \mathrm{~mm}$ ), with a $5 \mathrm{~m}$ receiver height (i.e., reflections occurring until $56 \mathrm{~m}$ from the receiver).

- with regards to the plane and ellipsoid approximations, differences are bigger than between the plane and sphere approximations when reflections occur farther than $550 \mathrm{~m}$ from the receiver. For farther reflections, differences between planes and ellipsoids become smaller than between planes and spheres. 
- the angular refraction due to troposphere can be negligible with regards to the position of the specular reflection point when the receiver height is below $5 \mathrm{~m}$, but is absolutely mandatory otherwise, particularly for satellites with low elevation angles where the correction to apply is exponential.

As a final remark, it is worth reminding the reader that the farther the specular reflection point is from the receiver, the more important the influence of the different error sources will be: Earth approximation, DEM integration, angular refraction. The farthest specular reflection points will be obtained for high receiver height and low satellite elevation. This simulator is likely to be of great help for the preparation of in situ experiments involving the GNSS-R technique. Further developments of the simulator will be implemented soon, such as a receiver installed on a moving platform in order to map the area covered by airborne GNSS-R measurement campaigns and on-board a LEO satellite.

Acknowledgements. This work was funded by CNES in the framework of the TOSCA project "Hydrologie, Océanographie par Réflectométrie GNSS (HORG)" and by the RTRA STAE foundation in the framework of the "Potentialites de la Réflectométrie GNSS In Situ et Mobile (PRISM)" project. Nicolas Roussel is supported by a $\mathrm{PhD}$ granted from the French Ministère de l'Enseignement Supérieur et de la Recherche (MESR).

Edited by: R. Marsh

\section{References}

Beckmann, P. and Spizzichino, A.: Scattering of Electromagnetic Waves from Rough Surfaces, Artech House Publishers, ISBN 089006-238-2, 1987.

Billich, A. L.: Improving the Precision and Accuracy of Geodetic GPS: Applications to Multipath and Seismology, PhD. B.S., University of Texas at Austin, M.S., University of Colorado, 2004.

Boehm, J., Niell, A., Tregoning, P., and Schuh, H.: Global Mapping Function (GMF): A new empirical mapping function based on numerical weather model data, Geophys. Res. Lett., 33, L07304, doi:10.1029/2005GL025546, 2006a.

Boehm, J., Werl, B., and Schuh, H.: Troposphere mapping functions for GPS and very long baseline interferometry from European Centre for Medium-Range Weather Forecasts operational analysis data, J. Geophys. Res. Sol.-Earth, 111, B02406, doi:10.1029/2005JB003629, 2006.

Cardellach, E., Fabra, F., Rius, A., Pettinato, S., and Daddio, S.: Characterization of Dry-snow Sub-structure using GNSS Reflected Signals, Remote Sens. Environ., 124, 122-134, 2012.

Chen, G. and Herring, T.: Effects of atmospheric azimuthal asymmetry on the analysis of space geodetic data, J. Geophys. Res. Sol.-Earth, 102, 20489-20502, doi:10.1029/97JB01739, 1997.

Géegout, P., Biancale, R., and Soudarin, L.: Adaptive Mapping Functions to the azimuthal anisotropy of the neutral atmosphere, J. Geodesy., 85, 661-667, 2011.
Gleason, S.: Remote Sensing of Ocean, Ice and Land Surfaces Using Bistatically Scattered GNSS Signals From Low Earth Orbit, Thesis (Ph.D.), University of Surrey, 2006.

Gleason, S., Lowe, S., and Zavorotny, V.: Remote sensing using bistatic GNSS reflections, GNSS Applications and methods, 399-436, 2009.

Helm, A.: Ground based GPS altimetry with the L1 openGPS receiver using carrier phase-delay observations of reflected GPS signals, Thesis (Ph.D.), Deutsches GeoForschungsZentrum (GFZ), 164 pp., 2008.

Hobiger, T., Ichikawa, R., Takasu, T., Koyama, Y., and Kondo, T.: Ray-traced troposphere slant delays for precise point positioning, Earth Planet. Space, 60, e1-e4, 2008.

Jarvis, J., Reuter, H., Nelson, A., and Guevara, E.: Hole-filled SRTM for the globe, CGIAR-CSI SRTM $90 \mathrm{~m}$ DAtabase, Version 4, CGIAR Consort for Spatial Inf., 2008.

Katzberg, S., Torres, O., Grant, M. S., and Masters, D.: Utilizing calibrated GPS reflected signals to estimate soil reflectivity and dielectric constant: results from SMEX02, Remote Sens. Environ., 100, 17-28, 2006

Komjathy, A., Zavorotny, V., Axelrad, P., Born, G., and Garrison, J.: GPS signal scattering from sea surface, Wind speed retrieval using experimental data and theoretical model, Remote Sens. Environ., 73, 162-174, 2000

Kostelecky, J., Klokocnik, J., and Wagner, C. A.: Geometry and accuracy of reflecting points in bistatic satellite altimetry, J. Geodesy, 79, 421-430, doi:10.1007/s00190-005-0485-7, 2005.

Lagler, K., Schindelegger, M., Boehm, J., Krsn, H., and Nilsson, T.: GPT2: Empirical slant delay model for radio space geodetic techniques, Geophys. Res. Lett., 40, 1069-1073, doi:10.1002/grl.50288, 2013.

Larson, K. M. and Nievinski, F. G.: GPS snow sensing: results from the EarthScope Plate Boundary Observatory, GPS Solut., 17, 4152, doi:10.1007/s10291-012-0259-7, 2013.

Löfgren, J. S., Rüdiger, H., and Scherneck, H. G.: Sea-Level analysis using 100 days of reflected GNSS signals, Proceedings of the 3rd International Colloquium - Scientific and Fundamental Aspects of the Galileo Programme, 31 August-2 September 2011, Copenhagen, Denmark, (WPP 326) 5 pp., 2011.

Lowe, S. T., Zuffada, C., Chao, Y., Kroger, P., Young, L. E., and LaBrecque, J. L.: 5-cm-Precision aircraft ocean altimetry using GPS reflections, Geophys. Res. Lett., 29, 1375, doi:10.1029/2002GL014759, 2002.

Marini, J. W.: Correction of satellite tracking data for an arbitrary tropospheric profile, Radio Sci., 7, 223-231. doi:10.1029/RS007i002p00223, 1972.

Martin-Neira, M.: A passive reflectometry and interferometry system (PARIS): Application to ocean altimetry, ESA J-Eur. Space Agen., 17, 331-355, 1993.

Nafisi, V., Urquhart, L., Santos, M. C., Nievinski, F. G., Bohm, J., Wijaya, D. D., Schuh, H., Ardalan, A. A., Hobiger, T., Ichikawa, R., Zus, F., Wickert, J., and Gegout, P.: Comparison of Ray-Tracing Packages for Troposphere Delays, Geosci. Remote Sens., 50, 469-481, doi:10.1109/TGRS.2011.2160952, 2012.

NASA and NIMA: The Development of the Joint NASA GSFC and the National Imagery and Mapping Agency (NIMA) Geopotential Model EGM96, NASA/TP-1998-206861, 1998.

Niell, A.: Preliminary evaluation of atmospheric mapping functions based on numerical weather models, Proceedings of the First 
COST Action 716 Workshop Towards Operational GPS Meteorology and the Second Network Workshop of the International GPS Service (IGS), 26, 475-480, 2001.

Nievinski, F. G. and Santos, M. C.: Ray-tracing options to mitigate the neutral atmosphere delay in GPS, Geomatica, 64, 191-207, 2010.

NIMA: National Imagery and Mapping Agency: Departement of Defense Wolrd Geodetic System 1984. NIMA Stock No. DMATR83502WGS84, NSN 7643-01-402-0347, 1997.

Nocedal, J. and Wright, S. J.: Numerical Optimization, Springer, ISBN 978-0-387-30303-1, USA (TB/HAM), 2006.

Rius, A., Aparicio, J. M., Cardellach, E., Martin-Neira, M., and Chapron, B.: Sea surface state measured using GPS reflected signals, Geophys. Res. Lett., 29, 37-1-37-4, doi:10.1029/2002GL015524, 2002.

Rius, A., Noque's-Correig, O., Ribo, S., Cardellach, E., Oliveras, S., Valencia, E., Park, H., Tarongi, J. M., Camps, A., Van Der Marel, H., Van Bree, R., Altena, B., and Martin-Neira, M.: Altimetry with GNSS-R interferometry: first proof of concept Experiment, GPS Solutions, 16, 231-241, doi:10.1007/s10291-0110225-9, 2012.

Rodriguez, E., Morris, C. S., Belz, J. E., Chapin, E. C., Martin, J. M., Daffer, W., and Hensley, S.: An assessment of the SRTM topographic products, Technical Report D-31639, JPL/NASA, 2005

Rodriguez-Alvarez, N., Bosch-Lluis, X., Camps, A., Vall-Llossera, M., Valencia, E., Marchan-Hernandez, J. F., and Ramos-Perez, I.: Soil moisture retrieval using GNSS-R techniques: Experimental results over a bare soil field, IEEE Trans. Geosci. Remote Sens., 47, 3616-3624, 2009.
Rodriguez-Alvarez, N., Camps, A., Vall-Llossera, M., BoschLluis, X., Monerris, A., Ramos-Perez, I. Valencia, E., MarchanHernandez, J. F., Martinez-Fernandez, J., Baroncini-Turricchia, G., Pérez-Gutiérrez, C., and Sanchez, N.: Land Geophysical Parameters Retrieval Using the Interference Pattern GNSS-R Technique, IEEE Trans. Geosci. Remote Sens., 49, 71-84, 2011.

Ruffini, G., Soulat, F., Caparrini, M., Germain, O., and MartinNeira, M.: The Eddy Experiment: Accurate GNSS-R ocean altimetry from low altitude aircraft, Geophys. Res. Lett., 31, L12306, doi:10.1029/2004GL019994, 2004.

Semmling, A. M., Beyerle, G., Stosius, R., Dick, G., Wickert, J., Fabra, F., Cardellach, E., Ribo, S., Rius, A., Helm, A., Yudanov, S. B., and d'Addio, S.: Detection of Arctic Ocean tides using interferometric GNSS-R signals, Geophys. Res. Lett., 38, L04103, doi:10.1029/2010GL046005, 2011.

Soulat, F., Caparrini, M., Germain, O., Lopez-Dekker, P., Taani, M., and Ruffini, G.: Sea state monitoring using coastal GNSS-R, Geophys. Res. Lett., 31, L21303, doi:10.1029/2004GL020680, 2004.

Treuhaft, P., Lowe, S., Zuffada, C., and Chao, Y.: 2-cm GPS altimetry over Crater Lake, Geophys. Res. Lett., 28, 4343-4436, 2004.

Wagner, C. and Klokocnik, C.: The value of ocean reflections of GPS signals to enhance satellite altimetry: data distribution and error analysis, J. Geodesy, 77, 128-138, doi:10.1007/s00190002-0307-0, 2003.

Zavorotny, A. U. and Voronovich, A. G.: Scattering of GPS signals from the ocean with wind remote sensing application, IEEE Trans. Geosci. Remote Sens., 38, 951-964, 2000.

Zus, F., Bender, M., Deng, Z., Dick, G., Heise, S., Shang-Guan, M., and Wickert, J.: A methodology to compute GPS slant total delays in a numerical weather model, Radio Sci., 47, RS2018, doi:10.1029/2011RS004853, 2012. 